\title{
机床智能控制系统体系架构及关键技术研究进展
}

\author{
孟博洋 $^{1}$ 李茂月 ${ }^{1}$ 刘献礼 $^{1}$ WANG Lihui ${ }^{2}$ \\ LIANG S Y ${ }^{3}$ 王志学 ${ }^{1}$ \\ (1. 哈尔滨理工大学切削加工及制造智能化技术教育部重点实验室 哈尔滨 150080 ; \\ 2. 瑞典皇家理工学院 斯德哥尔摩 瑞典 25175; \\ 3. 佐治亚理工学院乔治·W·伍德拉夫机械工程学院 亚特兰大 美国 30332)

\begin{abstract}
摘要: 机床智能控制系统是未来智能机床领域的重要组成部分, 对提高制造业核心竞争力具有重要意义。相比传统数控系统, 机床智能控制系统具有更高效、稳定地加工质量, 以及可代替人工经验智能判断等优点。针对现有机床智能控制系统方面的 综述性讨论较少的情况, 通过分析机床控制系统发展历程中四个阶段的特点, 提出机床控制系统的智能化体系和架构。然后, 从先进技术角度, 详细阐述了人工智能技术、数字孪生技术以及云服务等关键技术, 在机床智能控制系统中的应用。最后, 通过分析智能机床面临的几大严峻挑战与应对之策, 展望了未来机床智能化控制系统的发展趋势。
\end{abstract}

关键词: 数控机床; 智能控制系统; 人工智能; 数字孪生; 云服务

中图分类号: TG659

\section{Research Progress on the Architecture and Key Technologies of Machine Tool Intelligent Control System}

\author{
MENG Boyang $^{1} \quad$ LI Maoyue $^{1} \quad$ LIU Xianli $^{1} \quad$ WANG Lihui ${ }^{2}$ \\ LIANG S Y ${ }^{3}$ WANG Zhixue ${ }^{1}$
}

(1. Key Lab of Intelligent Technology for Cutting and Manufacturing, Ministry of Education, Harbin University of Science and Technology, Harbin 150080;

2. KTH Royal Institute of Technology, Stockholm, 25175 Sweden;

3. George W. Woodruff School of Mechanical Engineering, Georgia Institute of Technology, Atlanta, 30332 USA)

\begin{abstract}
Machine tool intelligent control system, as an integral part of future intelligent machine tools, plays a significant role in improving core competencies of manufacture. Compared with traditional CNC system, the intelligent one has the advantage of higher efficiency and more stable manufacturing quality. It's able to make intelligent decisions and substitute experiences of human operators. Considering that there are few reviews on intelligent control system of machine tools, the framework and architecture of the machine tool intelligent control system is proposed by analyzing features from four stages of historical development of the machine tool control system. From the perspective of advanced technology, the related key technologies and engineering applications are elaborated, such as artificial intelligence, digital twins and cloud services. At last, after analyzing several major challenges of intelligent machine tools and their countermeasures, the future development trend of machine tool intelligent control system is forecasted.
\end{abstract}

Key words: CNC machine tool; intelligent control system; artificial intelligence; digital twin; cloud service

\section{0 前言}

在全球制造业新一轮技术革新浪潮下，智能制
造是当今制造业的主导趋势 ${ }^{[1-2]}$ 。目前世界各国都在 采取积极行动, 以促进制造业在智能制造、智慧化 生产等领域的创新发展。美国出台 “国家先进制造 战略计划”、“先进制造伙伴计划”, 德国提出 “工

* 国家自然科学基金国际合作与交流重点资助项目(51720105009)。20200622

收到初稿, 20200906 收到修改稿 
业 4.0 战略计划”, 日本发布 “工业价值链参考框 架(IVRA)”，英国提出 “英国工业 2050 战略”、 法国提出 “新工业法国计划” 、韩国提出 “制造业 创新 3.0 计划”。美国布鲁金斯学会在 2018 年发布 的全球制造业评估报告中明确指出, 中国是国际制 造业的第一产出大国。目前, 我国在促进高端装备 制造业发展方面, 连续出台 “中国制造 2025、新一 代人工智能发展规划、国家智能制造标准体系建设 指南” 等指导政策, 旨在提高工业制造业智能控制 技术，加快制造业从自动化、信息化向智能化发展。

机床作为制造业的工业母机, 其产品占制造业 总量的 $40 \% \sim 60 \%$, 是智能制造领域的核心竞争力。 据国家统计局数据显示, 2019 年国内数控机床产业 规模占全球比重约为 $31.5 \%$, 位居全球第二[3]。然 而高档数控机床厂家却集中在德国、美国、日本等 国家, 国内高档精密机床行业仍需 “爬坡过坎”。 机床是智能制造发展的关键突破口, 也成为各工业 发达国家的竞争焦点 ${ }^{[4-5]}$ 。

机床智能控制系统作为高档机床的核心技术, 负责自动采集加工过程反馈信息、建立理论模型, 并通过自修正、自适应等加工控制技术, 使得加工 精度和效率稳定保持在一定的误差安全范围内。机 床智能控制系统影响着机床的加工精度、执行效率、 核心功能、稳定程度等多个关键技术指标, 其性能 直接决定机床整机的技术水平。因此, 机床智能控 制系统是一个国家机床产业发展水平的重要标志, 也是当今机床智能加工技术发展的主导趋势。研究 与提高机床智能控制系统, 对现代智能制造发展创 新具有重要意义。

国内外众多高校、企业和研究中心, 均对机床 及智能制造专题进行了不同程度上的深入探索和综 述性研究 ${ }^{[6-8]}$ 。在智能制造技术方面, 2018 年周济院 士、李培根院士将智能系统与先进制造技术的发展 总结归纳为三个范式, 指出未来智能制造的发展趋 势是以 “人、信息、物理系统(HCPS)” 为核心的新 一代智能制造 ${ }^{[9]}$ 。2019 年加拿大工程院 WANG 综 述了智能制造中人工智能技术的演变发展 ${ }^{[10]}$, 指出 人工智能、云计算、大数据分析、物联网等技术, 是未来 5 20 年智能制造的发展机遇和挑战。2020 年, 刘强教授研究提出智能制造理论体系的总体架 构 $^{[11]}$ 。

在机床控制系统智能技术方面, 2015 年叶佩青 综述了数控技术的发展 ${ }^{[12]}$, 提出通过创建 APP 组件 式数控平台的体系结构, 来营造数控发展舞台、突 破数控发展瓶颈的策略。2018 年刘献礼综述了智能
技术在机床切削加工中的应用 ${ }^{[13]}$ 。黄荣等讨论了云 计算在智能机床控制体系中的应用，指出云计算对 机床智能系统的发展优势 ${ }^{[14]}$ 。2019 年华中数控公司 以华中 9 型智能数控系统(INC)为例, 提出基于新一 代人工智能的机床智能系统 ${ }^{[15]}$ 。总结从手动机床到 数控机床再到智能机床的发展历程, 明确指出智能 化控制系统是下一代智能机床发展方向。

机床的智能化发展离不开智能控制系统, 然而 针对机床智能控制系统方面的综述性讨论却较少。 本文将结合本领域国内外研究成果, 归纳机床控制 系统及智能技术的发展历程, 对新一代机床智能控 制系统的内涵、技术体系框架及主要研究方向进行 全面分析和总结。最后展望未来机床智能系统在工 程应用等方面的发展趋势和应用前景。机床智能控 制系统是高端装备制造业的发展核心，也是现代制 造业创新发展的先决条件, 机床智能控制系统的研 发对提高国家制造业核心竞争力具有重要意义。

\section{1 机床控制系统及智能技术的发展}

\section{1 机床控制系统及制造业演变历程}

数十年来, 机床行业的发展从传统的机械控制 系统、到数字化控制系统、再到自动化控制系统乃 至智能化控制系统, 经历了一系列的技术发展与革 新 ${ }^{[16-18]}$ 。同时, 随着制造业不断发展与技术革新, 机床及控制系统得到了快速发展, 众多先进的智能 技术也被提出和广泛应用 ${ }^{[19-20]}$ 。

面对不断涌现的新技术、新理念、新模式, 众 多专家、学者对机床及先进制造技术的发展不断进 行总结、归纳 ${ }^{[21]}$ 。奥克兰大学徐旬 ${ }^{[22]}$ 根据工业发展 的四个阶段特点, 将机床技术的发展对应划分为机 床 1.0、机床 2.0、机床 3.0、机床 4.0 四个阶段。华 中科技大学陈吉红 ${ }^{[15]}$ 根据智能制造的三个范式和 机床的发展历程, 将机床发展细化为手动机床、数 控机床、互联网+机床、智能机床四个阶段, 并详细 阐述了各时代机床中的发展差异。但在机床技术的 发展过程中, 较少单独对机床控制系统的发展阶段 进行划分与详细总结。机床控制系统技术发展演变 应与机床技术的革新历程同步。因此, 机床控制系 统的技术发展同样可以划分为四个主要发展阶段。 本文从机床控制系统的平台架构及智能技术发展的 角度出发, 将其归纳为机械控制架构、嵌入式架构、 扩展式架构、云架构四个阶段, 如图 1 所示。并且 进一步讨论各个不同时代机床控制系统与相关制造 模式的特点。 


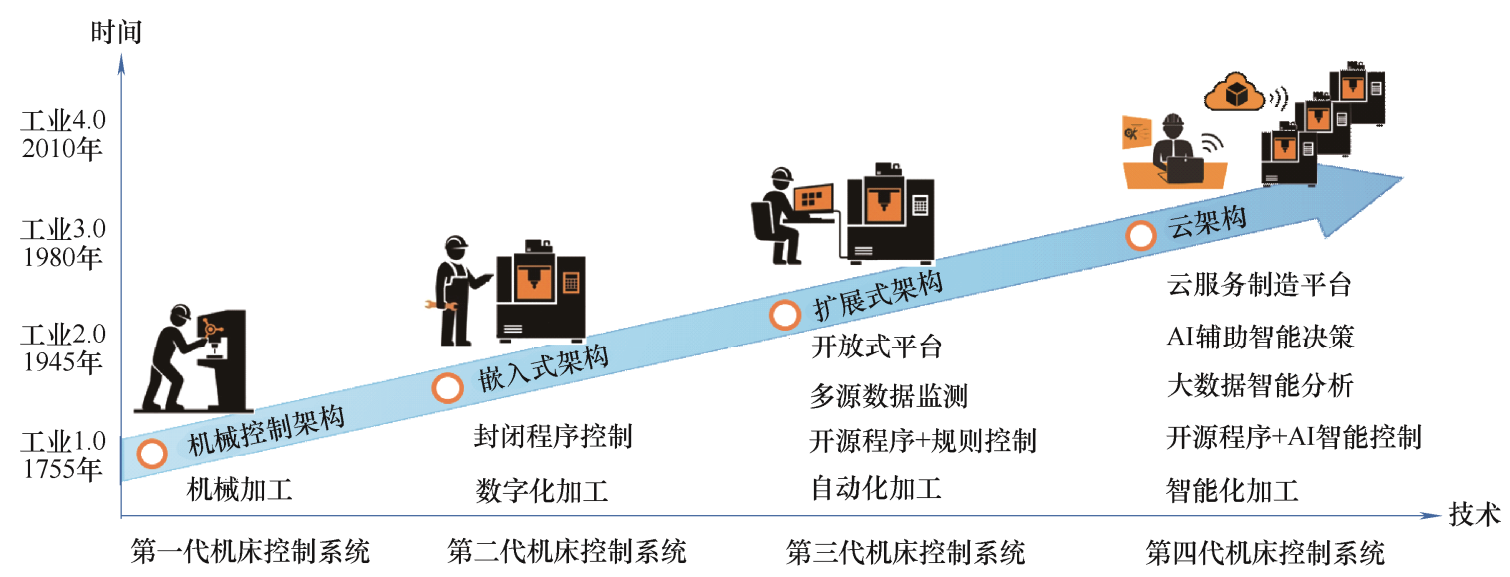

图 1 机床控制系统发展及演变历程

\section{2 机床控制系统的技术发展}

(1) 第一代机电控制系统。

20 世纪以前(始于约 1775 年), 机床采用机械结 构的控制系统。机床加工过程中的感知、分析和决 策依赖于工人操作经验, 产品质量不稳定, 正面临 被逐步淘汰的趋势。机电控制系统的主要特点是由 机器代替人力加工, 简化了生产与制造的流程。同 时, 手动机电控制信号的普及与规范化也为机床数 控系统提供了前置技术基础, 是典型的人与物理系 统之间的交互操作。

(2) 第二代嵌入式系统。

20 世纪中期(始于约 1945 年), 嵌入式架构作 为机床系统的典型代表, 是当时机床系统使用最 广泛的一种架构形式。嵌入式系统核心由专用嵌 入式芯片(如 ARM、FPGA 等)硬件控制, G 代码 作为机床控制语言规范开始普及, 克服了当时机 床控制对人工技术的依赖, 具有较好的稳定性。 但由于嵌入式系统的软硬件高度关联, 导致机床 系统开放性和灵活性不足。致使系统的开发周期 长、固件更新换代慢, 限制了其本身的持续开发 潜力。目前国内的嵌入式机床控制系统技术成熟, 控制精度、核心功能与国外嵌入式系统水平相当。 特别是在中小型及老品牌数控机床中应用较多, 如早期型号的日本 FANUC、德国 Siemens、 Heidenhain、法国 NUM、西班牙 FAGOR、国内 华中数控、沈阳数控、广州数控、北京凯恩帝等 数控系统。

(3) 第三代工控机系统。

20 世纪末期(始于约 1980 年), 计算机技术的迅 速提升, 推动了机床系统扩展式架构的诞生。扩展
式架构硬件基于工业计算机(IPC), 将机床控制器的 全部功能均由计算机软件实现, 通过统一的开发环 境(Windows/Linux 等), 使装在工控机上的各种软件 可以驱动机床的对应功能, 如人机交互接口(HMI)、 译码、插补、软 PLC 通讯、数据信号采集、实时分 析、运动控制等。扩展式系统架构的主要特点是将 计算机操作系统作为研发基础, 具有良好的兼容性 和通用性，解决了制造业市场频繁变换的个性化控 制需求与传统数控系统封闭式架构之间的矛盾冲 突，也使人、信息、物理系统的进一步交互更加 完善。

目前在第三代机床控制系统研发方面, 国内 数控系统技术与国外技术水平逐步接近。例如日 本 Mazak 基于 Windows 8 系统环境下开发的 MAZATROL Smooth 数控系统, 通信接口集成美 国 MTConnect 通用互联通信协议。德国 DMG MORI 提出的 CELOS 系统, 将机床环境和 PC 系 统一体化集成，并且兼容 PPS、ERP、CAD/CAM 等软件协作控制。华中数控 8 型 INC 系统可以与 微软的 Windows 系统融为一体, 实现数控系统与 $\mathrm{PC}$ 系统的无缝衔接。沈阳 $\mathrm{i} 5 \mathrm{OS}$ 数控系统将控制 核心进行模块化封装并提供标准的开发 API, 同 时支持 Linux、Windows、Android、IOS 等多平 台的 APP 开发环境。

(4) 第四代云系统。

21 世纪初期(始于约 2010 年), 基于云架构的机 床控制系统是未来研究热点和机床厂家争夺焦点。 机床云控制系统的主要特点是在硬件设备上集成了 服务器群组, 在软件架构上深度融合了人工智能为 代表的控制技术、工业物联网技术、云服务技术等。 
2015 年德国斯图加特大学在云计算的基础上提出 “全球本地化” 云端数控系统, 将传统数控系统的 人机界面、数控核心和 PLC 都移至云端, 本地仅保 留机床的伺服驱动和安全控制, 在云端增加通信模 块、中间件和以太网接口, 通过路由器与本地数控 系统通信。

与传统的机床控制系统相比, 云架构机床系 统采用的服务器群组的集成方式使得神经网络等 智能算法的训练和计算得到极大加强, 云数据服 务可以对大数据分析提供更好的信息支持, 云上 位控制编程处理灵活、开放性更彻底，同时支持 手机、笔记本、工业计算机、虚拟现实等多种人 机交互前端，是机床控制系统发展的重要方向。 目前有许多机床企业已在云服务方面开展研究, 如 Mazak 公司提出的 iCONNECT、FANUC 公司 的 FIELD system, DMG MORI 公司提出的 TULIP、华中数控的 iNC-Cloud 云管家, 沈阳机 床的 iSESOL 云平台等。目前机床控制系统各发 展阶段的主要特点如表 1 所示。

表 1 机床控制系统各阶段发展特点

\begin{tabular}{|c|c|c|c|}
\hline 机床系统 & 第二代 & 第三代 & 第四代 \\
\hline 系统架构 & 嵌入式 & 工控扩展式 & 云架构 \\
\hline 系统平台 & 嵌入式平台 & PC 系统平台 & $\mathrm{PC}$ 平台 + 云平台 \\
\hline 核心设备 & $\begin{array}{c}\text { 专用微处理 } \\
\text { 器 }\end{array}$ & 工控机 & $\begin{array}{c}\text { 工控机和服务器 } \\
\text { 群 }\end{array}$ \\
\hline 开发环境 & $\begin{array}{c}\text { 嵌入式 } \\
\text { 开发环境 }\end{array}$ & $\mathrm{PC}$ 系统环境 & $\begin{array}{c}\text { PC 系统环境 } \\
\text { +云服务器环境 }\end{array}$ \\
\hline 软件平台 & 嵌入式系统 & $\begin{array}{c}\text { PC 系统+专家 } \\
\text { 系统 } \\
\text { +数据库+网络 } \\
\text { 通信 }\end{array}$ & $\begin{array}{c}\mathrm{PC} \text { 系统+数字孪 } \\
\text { 生 } \\
\text { 平台 }+ \text { 大数据平台 } \\
+ \\
\text { 人工智能平台 }+ \text { 云 } \\
\text { 端 }\end{array}$ \\
\hline $\mathrm{CNC}$ 软件 & $\begin{array}{c}\text { 集成固件升 } \\
\text { 级 }\end{array}$ & $\mathrm{App}+$ 模块升级 & App+云推送 \\
\hline 数据接口 & $\begin{array}{c}\text { 专用芯片接 } \\
\text { 口 }\end{array}$ & 板卡 PCI 等接口 & 工业以太网接口 \\
\hline $\begin{array}{c}\text { 外部信息共 } \\
\text { 享 }\end{array}$ & 信息孤岛 & 本地+网络共享 & 本地+云端共享 \\
\hline $\begin{array}{l}\text { 支持共享的 } \\
\text { 数据信息 }\end{array}$ & $\begin{array}{c}\mathrm{G} \text { 代码、 } \\
\mathrm{DNC} 、 \mathrm{I} / \mathrm{O} \\
\text { 等 } \\
\text { 少量控制信 } \\
\text { 号 }\end{array}$ & $\begin{array}{l}\text { 支持译码、通信、 } \\
\text { 多源感知数据、 } \\
\text { 加工过程监控、 } \\
\text { 规则决策、健康 } \\
\text { 监控等多个模块 } \\
\text { 数据协作 }\end{array}$ & $\begin{array}{c}\text { 在本地模块协作 } \\
\text { 的基础上, 支持云 } \\
\text { 端 CAD } \backslash \mathrm{CAM、} \text { 、 } \\
\mathrm{EMS} 、 \mathrm{ERP} 、 \text { 售后 } \\
\text { 服务等全生命周 } \\
\text { 期数据管理 }\end{array}$ \\
\hline 数据来源 & $\begin{array}{c}\text { 内部封闭数 } \\
\text { 据 }\end{array}$ & 多源数据 & 大数据 \\
\hline $\begin{array}{l}\text { 其他设备 } \\
\text { 的协作性 }\end{array}$ & 设备级协作 & 车间级协作 & 企业级协作 \\
\hline 网速需求 & 无 & 低速 & 高速 \\
\hline 系统开放性 & 低 & 中 & 高 \\
\hline
\end{tabular}

\section{2 机床智能控制系统技术体系架构}

\section{1 机床智能控制系统的内涵及特点}

机床控制系统的本质是指对机床生产加工进行 控制的操作设备。目前有很多学者提出对智能机床 的理解和看法 ${ }^{[9,15]}$, 然而对于机床智能控制系统的 详细定义和观点较少。因此对机床智能控制系统的 内涵、特点与理解，一直是学术关注的焦点。本文 参考专家学者对智能机床以及智能制造的观点与看 法，提出对机床智能控制系统的理解。

机床智能控制系统应符合新一代智能制造中 “人-信息-物理系统” 的核心范式和层级体系理 论。同时，也应符合智能机床特性中的 “智能” 体现，包括 “感知、分析、决策、执行” 等过程 中的智能思考、自我学习等自主独立的思维特性。 在此基础上，还应满足机床控制系统的发展历程 及技术演变趋势。例如, 更加大量、复杂的数据 处理能力, 更加便捷、准确的闭环分析与控制体 系，更加泛用、兼容的协同工作架构等。最终， 通过不断引入各种先进的控制技术，革新智能的 硬件设备以及对控制体系框架的不断改进，达到 机床控制系统向智能化的跃进，即机床智能控制 系统。

\section{2 机床智能控制系统体系架构}

随着科学技术的发展, 人们对机床智能控制系 统认识是一个不断发展、逐步深化的过程。从传统 的单一控制器到如今的工控机和多服务器群组, 从 传统的单一目标控制, 到如今庞大的集群综合管控, 机床智能控制系统的体系架构也越来越复杂。当前 迫切需要在总结过去机床控制系统发展历史、理论 和技术研究成果的基础上，形成一个新的机床智能 控制系统体系架构。对其体系架构的研究可以从硬 件框架以及软件体系两方面分析。

\subsection{1 机床智能控制系统的物理平台框架}

机床智能控制系统的物理平台是指组成机床 智能机床控制系统在运行时的必要设备组件。除 直接参与机床智能控制运算的工控机、服务器群 组等必要设备外，同时也包含辅助机床智能控制 系统的智能组件、智能传感器、云平台以及物联 网等设备。

机床智能控制系统的物理平台框架的合理性, 直接影响到机床智能控制系统在运行时的稳定程 
度、核心功能、执行效率等关键技术指标。因此, 物理平台在构建时的功能规划、框架划分的合理性、 科学性以及正确性, 是构建机床智能控制系统时需
要考量的首要因素。本文提出的机床智能控制系统 的物理平台框架如图 2 所示, 该物理平台框架主要 由以下几个部分组成:
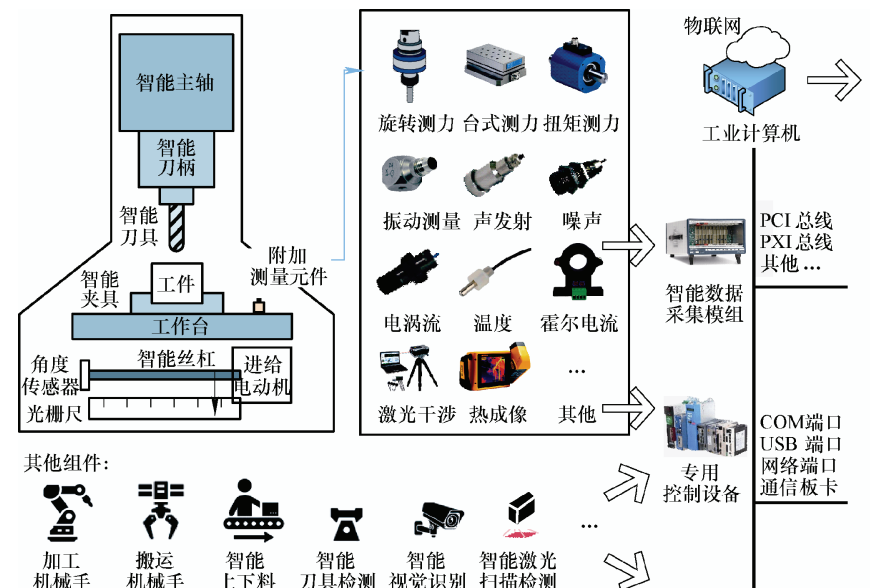

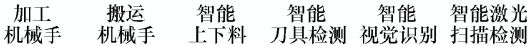

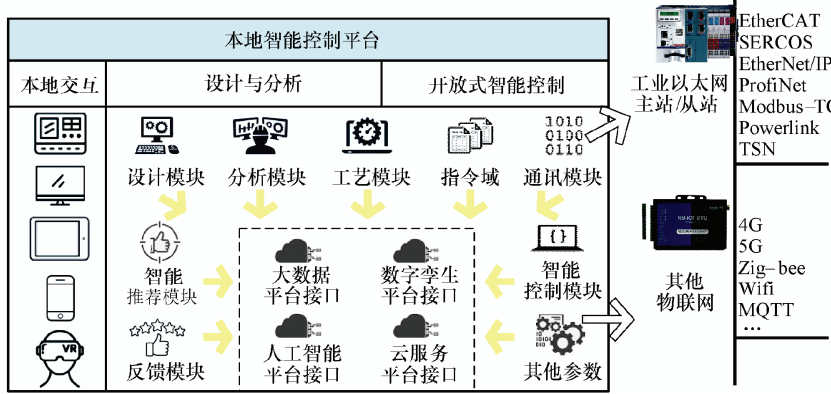

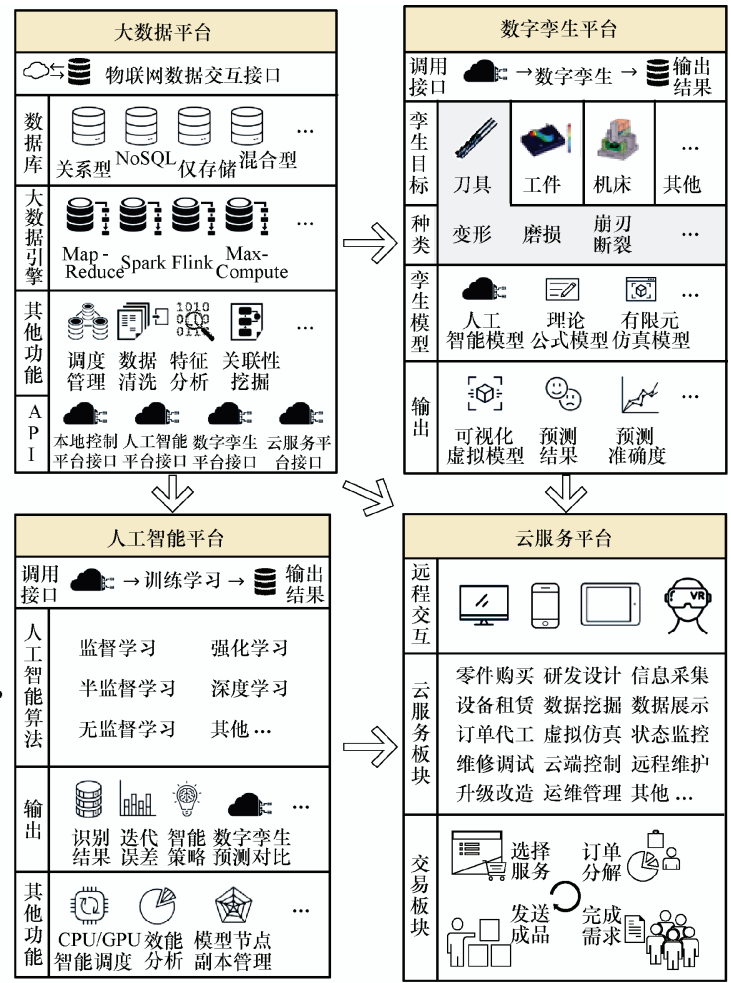

图 2 机床智能控制系统的物理平台框架

(1) 智能感知元件。

机床智能控制系统依赖于感知元件所反馈的数 据, 来判断当前系统所处的加工状态。因此, 大量 的感知元件的引入是机床智能控制系统物理平台的 组成特点之一，如表 2 所示。

表 2 机床智能控制系统的感知信息及感知元件

\begin{tabular}{|c|c|c|}
\hline $\begin{array}{l}\text { 数据 } \\
\text { 结构 }\end{array}$ & 类别 & 感知元件 \\
\hline \multirow{5}{*}{$\begin{array}{l}\text { 结构 } \\
\text { 化数 } \\
\text { 据 }\end{array}$} & 力 & $\begin{array}{l}\text { 旋转式测力仪、台式测力仪、扭矩传 } \\
\text { 感器、压力传感器、应变片、智能主轴、 } \\
\text { 智能刀柄、智能刀具、智能丝杜 }\end{array}$ \\
\hline & 振动 & $\begin{array}{l}\text { 振动传感器、 } \mathrm{AE} \text { 声发射、噪音传感器、 } \\
\text { 智能刀柄、智能刀具 }\end{array}$ \\
\hline & 位置 & $\begin{array}{l}\text { 光栅尺、编码器、球杆仪、 } \\
\text { 电浴流传感器、激光干涉仪、探针、 } \\
\text { 智能主轴、智能刀柄、智能丝杜 }\end{array}$ \\
\hline & 温度 & $\begin{array}{l}\text { 热电偶式温度传感器、辐射式温度传 } \\
\text { 感器、智能主轴、智能丝杜、智能刀具 }\end{array}$ \\
\hline & 能耗 & $\begin{array}{l}\text { 霍尔传感器、电流电压表、 } \\
\text { 功率计、智能主轴 }\end{array}$ \\
\hline \multirow{2}{*}{$\begin{array}{l}\text { 非结 } \\
\text { 构化 } \\
\text { 数据 }\end{array}$} & $\begin{array}{l}\text { 视频 } \\
\text { 图像 }\end{array}$ & 工业摄像头、 CCD 相机、热像仪 \\
\hline & $\begin{array}{c}\text { 三维 } \\
\text { 模型 } \\
\end{array}$ & 激光扫描仪 \\
\hline $\begin{array}{l}\text { 半结 } \\
\text { 构化 } \\
\text { 数据 }\end{array}$ & $\begin{array}{l}\mathrm{G} \text { 代码、XML } \\
\text { 信息表、PLC 逻 } \\
\text { 辑图 }\end{array}$ & 专用物联网设备 \\
\hline
\end{tabular}

随着科学技术的发展, 机床智能控制系统的 感知元件也发生了较大的变化和技术革新。目前 机床智能控制系统智能感知元件主要有以下 三类。

1) 附加测量元件。附加测量元件是指机床外部 附加的可拆卸类传感器, 如测力仪、声发射传感器、 激光干涉仪等。该类智能感知元件的特点是与机床 智能控制系统的弱相关性。附加测量元件一般具有 较高的测试精度, 可以根据系统的感知需求较为随 意的增减, 适合于实验、测试等中短期的感知需求 中使用。

2) 智能组件。智能组件是在传统的机床组件基 础上融入传感器, 从而形成的新一代感知元件, 如 智能主轴、智能刀具等。该类智能感知元件结合了 信息感知能力与机床传统组件的优点。由于可以较 为直接的接触被测变量, 敏感性一般较高。既适合 在高档机床的生产加工中长期使用, 也可以进行适 当的拆卸更换。

3) 传统执行类组件。传统执行类组件是指在 机床智能控制系统中执行控制命令的一类组件, 
例如进给伺服电机等。通过结合开放式架构的机 床智能控制系统, 可以截获并反馈系统中的控制 命令。因此, 该类智能感知元件既可以是执行机 构, 也可以当成感知元件使用。该类智能感知元 件仅需要机床智能控制系统的软件支持, 成本较 低。但由于不是专业设计用于信息反馈, 测量精 度往往较低。仅适合精度需求较低且长期使用的 工况。

(2) 工业云平台。

目前学术研究中工业云平台的概念尚未明确统 一, 本文中的工业云平台是指集成了大数据平台、 数字孪生平台、人工智能平台、云服务平台以及物 联网平台等多个平台的工业生态环境。工业云平台 是机床智能控制系统物理平台的重要组成部分, 也 是机床智能控制系统与传统数控系统在物理平台方 面的重要区别。

其中物联网平台, 负责将机床智能控制系统 中的感知数据以及其他系统信息, 采集并上传到 云端大数据平台中。物联网平台是机床智能控制 系统与工业云平台链接重要组成环节。因此, 对 于物联网平台的兼容性具有较高的要求。根据对 传输数据的速度以及实时性的不同要求, 物联网 应适配多数的工业通信协议, 如 EtherCAT、5G、 MQTT 等。大数据平台负责机床智能控制系统中 的数据储存环节。在普通的存储设备中, 当数据 量达到 TB 以上级别时, 数据的查询及调取往往 花费较长时间。而大数据平台通过将数据存储在 专用结构的数据库中, 同时结合大数据引擎的使 用, 可以大幅度缩短所需数据的查询及调取时 间。人工智能平台与数字孪生平台负责机床智能 控制系统中的强化计算与分析环节。其特点是可 以自由、弹性化的调用所需资源，如算力资源、 数据资源、模型及算法资源等。云服务平台负责 机床智能控制系统中的业务环节。云服务平台的 建立, 将传统机床中的技术价值向电子商务化转 变, 极大地加强了技术价值的流通。同时, 也使 得机床智能控制系统的服务范围快速扩张。

目前, 已有许多企业开展工业云平台生态环境 的探索研究。在物联网方面, FANUC 公司提出的 MT-LINKi 设备可以对该公司的 CNC 机床、机器人 控制器、支持 OPC-UA 通信的 PLC, 以及支持 MTConnect 通信的机床等设备进行信息采集。沈阳 机床厂提出的 ISESOL BOX 支持 FANUC、
SIEMENS、i5 等数控系统的物联网连接。在大数据 及人工智能方面, 国内已有较成熟的通用平台, 如 阿里云、百度云、浪潮云等。在数字孪生方面, 国 内多数企业仅提供虚拟建模支持, 复杂的孪生预测 模型涉及较少。在云服务平台方面，沈阳机床厂提 出的 iSESOL 平台中机床厂云服务功能较为丰富。

(3) 本地智能控制平台。

本地智能控制平台的设备多以工业计算机为 主，是机床智能控制系统中的控制主体。由于本地 智能控制平台硬件在机床控制命令传输中的优越 性, 也有部分学者将本地控制平台与物联网设备融 合。即使用本地工业计算机先与数据采集设备统一 链接, 然后再通过工业计算机将数据上传到大数据 平台的方法来取代物联网设备。

\subsection{2 机床智能控制系统的控制体系架构}

控制体系架构是指机床控制系统在软件方面各 组成模块的控制关系及层级结构。对控制体系架构 理论内涵的进一步理解，可以从以下两方面分析：

首先从控制体系的层级结构角度分析, 机床智 能控制系统的体系架构基于 HCPS 的理论体系和层 级框架。可以划分为数据感知层、建模分析层、智 能决策层、控制执行层四个主要层级组成。

其次，从机床加工流程的角度分析，机床智能 控制系统贯穿于机床零件制造的全部控制流程。根 据被加工零件生产的流程顺序，机床智能控制系统 可以划分为加工工艺的智能设计模块、加工状态的 智能感知与自主建模分析模块、加工过程的智能控 制模块、加工后大数据的自学习与优化模块四个主 要机床控制系统模块组成。

结合上述两方面的分析，梳理机床智能控制 系统的具体感知信息、模型分析、决策控制、内 部指令等环节流程。最终可以从软件控制体系方 面, 得到机床智能控制系统的控制体系架构, 如 图 3 所示。

\section{3 机床智能控制系统的关键技术}

\subsection{1 人工智能技术}

人工智能技术是人与信息系统的深度融合，更 是机床智能系统的 “控制核心” [23]。在机床智能系 统中人工智能技术体现在智能决策的自主性。如加 工工艺的智能推理制订, 加工信息的自主建模与智 能分析, 加工过程的智能决策、智能控制, 加工之 后工业大数据累积知识的自成长学习、智能迭代优 化等多个方面 ${ }^{[2]}$ 。 


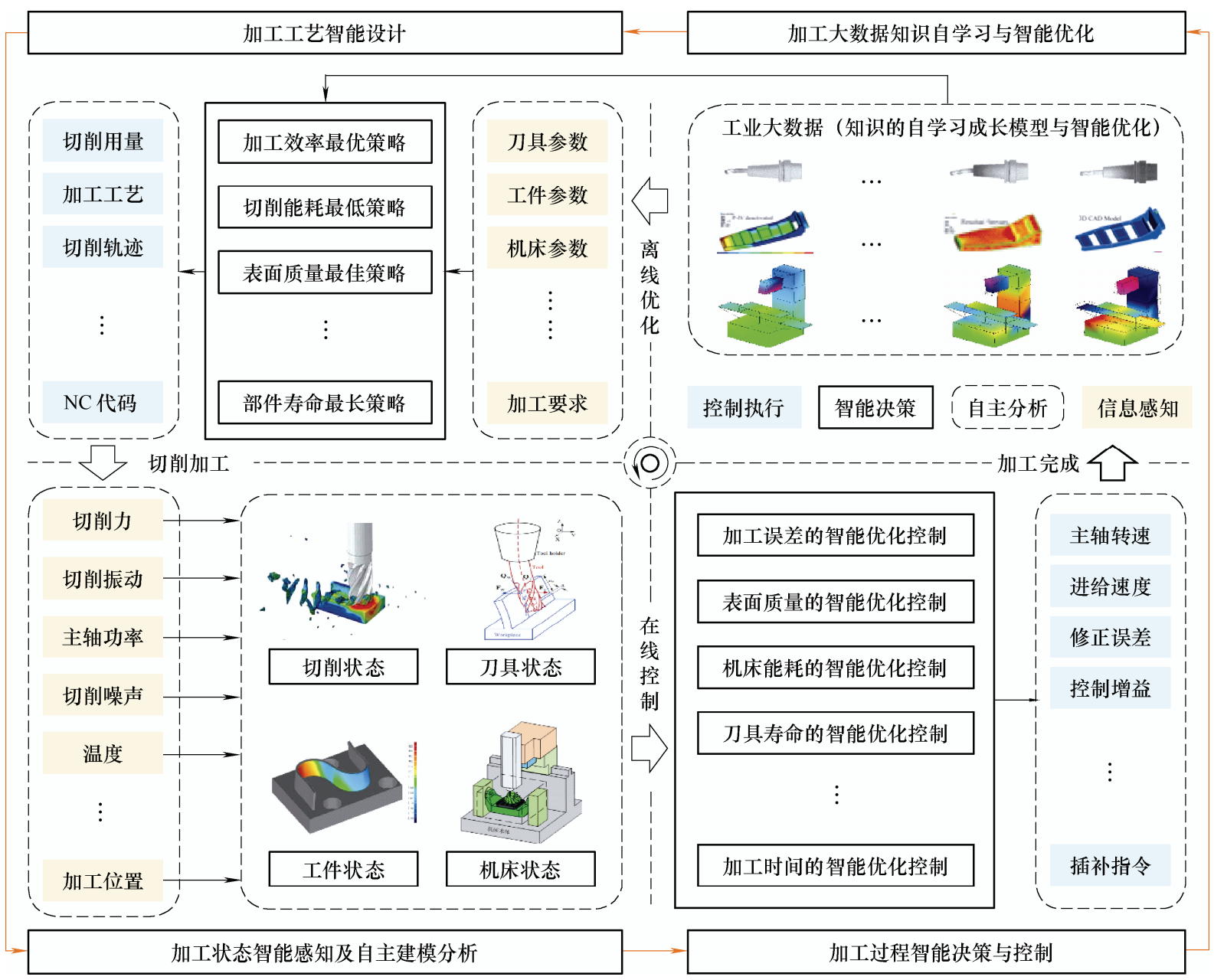

图 3 机床智能控制系统的控制体系架构

\subsection{2 数字孪生技术}

数字孪生技术是信息与物理系统的深度融合, 也是连接机床与传感器、工艺系统、加工协作信息、 大数据知识库等重要信息的 “交通枢纽” ${ }^{[25-26]}$ 。近 年来数字孪生技术也出现与人工智能技术和云技术 结合的发展趋势。有文献[27]将数字孪生中异构数 据获取和集成管理, 及数据的同步标签分析方法等 方面, 与人工智能技术交叉融合, 形成智能孪生的 趋势。也有文献[28]将数字孪生与云服务技术结合, 利用云数据上的优势形成基于云的数字孪生。

\subsection{3 云服务技术}

云服务技术是人与物理系统的深度融合, 也是 支持机床智能系统工作的 “基础平台”。云服务将本 地物理系统、本地作业建立网络对外接口, 向服务 化转变。如人机交互平台服务化、控制软件服务化、 加工流程服务化、数据孪生服务化等, 是制造业与 服务业融合的新形态 ${ }^{[29]}$ 。

\section{3 机床智能控制系统的人工智能技术}

人工智能技术在机床智能系统中的应用贯穿于
整个切削加工过程，包括加工前、加工中、加工后 三个部分 ${ }^{[30-31]}$ 。人工智能技术具有代替人工进行感 知、分析、推理、决策的优势，可以大幅度提高机 床控制系统的智能水平。围绕对应三个加工过程, 前期工作已经讨论了加工过程的智能技术 ${ }^{[13]}$ 。因 此, 本节重点从加工前工艺的智能推理决策、加工 后大数据知识的智能自学习复用两个方面, 详细介 绍人工智能技术在机床智能系统中的应用方式, 并 对其主要特点进行剖析。

\section{1 加工前工艺的智能决策}

加工前工艺的智能决策是指零件在加工前进行 的一系列复杂工艺任务流程, 包括零件要求分析、

工艺方法选择、加工特征排序、加工轨迹规划、加 工过程仿真以及 $\mathrm{NC}$ 程序智能编制等多范围、多领 域的任务知识 ${ }^{[32]}$ 。传统的工艺流程由设计人员、工 艺人员、调度人员、生产人员等一人或多人协作完 成, 员工依赖于自身的经验和知识对刀具、加工轨 迹、切削用量等参数进行人工拟定。而当前机床智 能系统正在从单一的加工设备逐渐转变为包含设 计、仿真、工艺、生产加工及售后服务等一体化的 集合体。工艺的智能决策是机床智能系统将一个零 
件从设计模型转化为加工成品时, 需要进行的首要 步骤, 也是连接设计人员与制造产品之间的重要 桥梁。

\subsection{1 工艺方法的智能决策}

工艺方法智能决策的第一个基础环节是将加工 工件从 $\mathrm{CAD}$ 模型转化为工艺知识。其中包括对零 件特征的智能分析与识别, 然后根据零件的特征类 型、尺寸、工艺要求等加工信息智能决策出最佳的 工艺方法。JING 等 ${ }^{[33]}$ 基于工艺知识提出一种系统化 的工艺智能规划方法。该方法对零件从特征识别分 析, 到工序的智能决策进行了系统化的智能决策方 法集成。

张禹等 ${ }^{[34]}$ 针对宏观工艺决策, 基于改进的 $\mathrm{BP}$ 神经网络, 提出了面向 STEP-NC 2.5D 制造特征的 智能宏观工艺规划方法(图 4)。该方法建立了用于 STEP-NC 2.5D 制造特征加工操作方法决策的集成 BP 神经网络模型。通过结合混沌算法、遗传算法改 进了 BP 神经网络, 并提出零件加工信息的归一化处 理规则, 提高了宏观工艺规划的智能决策速度。

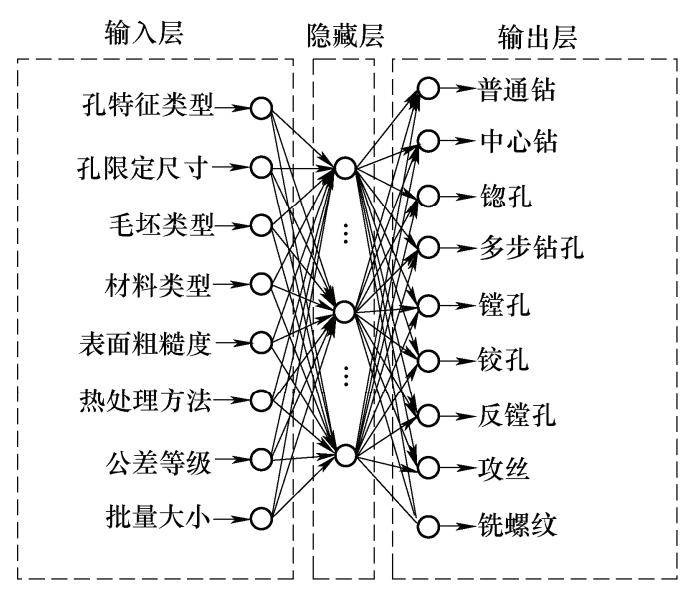

图 4 孔特征加工操作方法的 BP 神经网络决策 ${ }^{[34]}$

\subsection{2 工艺路线的自适应优化}

在加工及工艺规划的过程中, 系统的加工环境、 资源状况、加工情况等并非恒定不变。这时规划好 的工艺方法应能根据环境的变化, 进行自主决策和 判断最佳工艺路线。JI 等 ${ }^{[35]}$ 针对加工过程中刀具信 息可能发生意外改变的情况, 提出基于可达性的加 工特征路径的最佳排序方法。该方法将加工特征以 地图的形式映射, 并通过邻接矩阵和可达矩阵, 使 得刀具信息在发生意外改变时, 工艺系统可以做出 自适应决策, 以减少换刀次数, 并满足相应的加工 要求。

在不断变化的制造环境中，制造资源也存在许 多不确定因素。LIU 等 ${ }^{[36]}$ 针对大型零件的小批量制 造需要长时间的工艺规划情况, 将零件的加工特征
作为工艺规划知识的基础载体，提出了一种基于加 工特征和智能体的工艺路线自适应方法，如图 5 所 示。该方法在工艺方法决策阶段需要人工干预，不 能完全自动执行; 但突出的优点是当制造环境发生 变化时, 智能体可以实时感知制造资源的变化, 并 通过多个智能体之间的交流做出最佳工艺路线 决策。

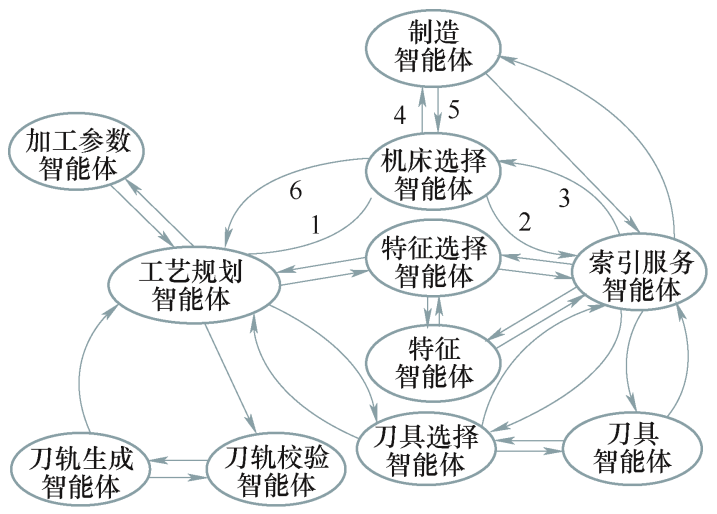

图 5 多个智能体协作工艺规划 ${ }^{[36]}$

除加工资源以及加工环境外，加工工步的工艺 参数以及加工先后顺序等导致的加工状态变化, 对 工艺路线的自适应决策也有着重要影响。图 6 所示 的五轴铣削加工过程, 分析了前一阶段加工策略的 选择对后续阶段的加工策略影响及选择。BUDAK 等 ${ }^{[37}$ 通过对粗加工、半精加工和精加工等阶段进行 过程建模和仿真分析，比较了不同加工策略以及加 工参数对颤振稳定性、切削转矩、轴功率、刀具变 形、表面粗糙度等的影响程度，并提出工艺路径策 略选择优劣的定量比较方法。采用的五轴复杂曲面 铣削试验, 验证了该工艺路线智能决策方法可在保 证加工质量的前提下，使机床的整体加工时间节省 约 $30 \%$ 。

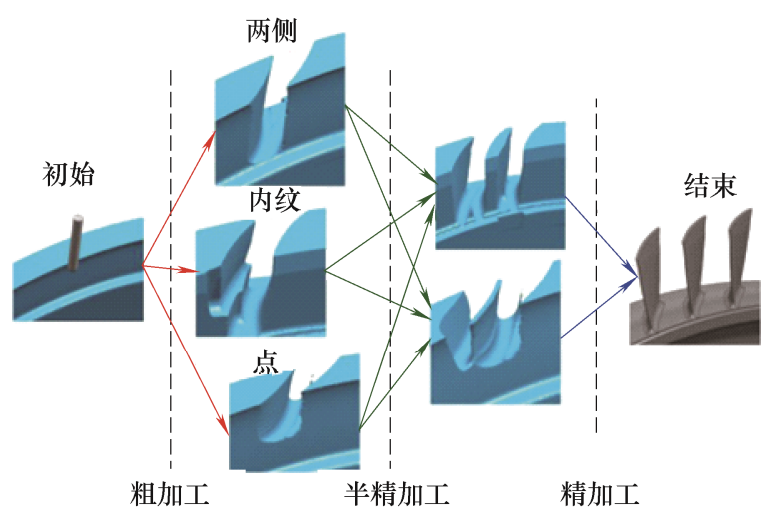

图 6 叶轮加工中典型的工艺路线动态策略选择 ${ }^{[37]}$

\subsection{3 加工轨迹的智能规划}

在加工工艺路线确定之后，机床智能控制系统 
需要针对工艺特点规划相应的加工轨迹 ${ }^{[38]}$ 。例如, 针对孔螺纹特征的加工轨迹智能规划问题。CHEN 等 ${ }^{[39]}$ 结合旅行商问题(TSP)和禁忌搜索(TS)算法两 阶段优化, 开发了一种加工轨迹的智能工艺规划 (i-CAPP), 并通过向 CNC 自动生成代码来进行加工 验证, 两种算法规划的轨迹如图 7 所示。

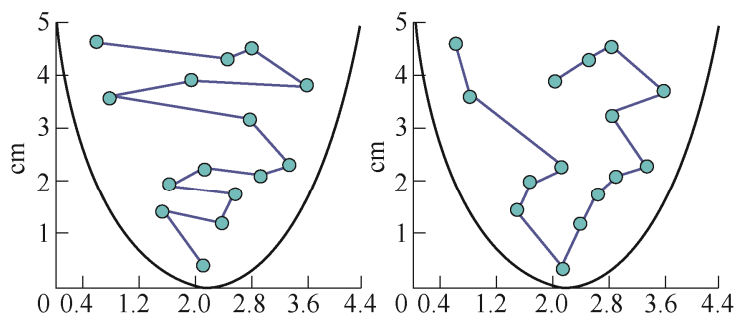

(a) zig-zag算法轨迹

(b) tabu算法轨迹

图 7 针对孔特征攻丝的轨迹智能规划 ${ }^{[39]}$

针对铣削刀具轨迹特征段的智能优化问题, KARUPPANAN 等 ${ }^{[40]}$ 提出一种元启发式优化求解方 法。该方法采用粒子群算法和遗传算法生成优化问 题的质量解。并将优化结果与 Autodesk Inventor HSM 和 Mastercam 工艺软件优化结果进行比较, 试 验表明所提出的优化方法, 可在加工过程中节省多 达 40\%的刀具空程。另外, 针对五轴加工中线性刀 路轨迹的不连续导致的振动及加工质量下降问题, 赵欢等 ${ }^{[41]}$ 采用 2 条 3 次 Bézier 曲线对线性刀路进行 转接光顺，同时结合前瞻技术与 7 段式 $\mathrm{S}$ 型速度规 划方法, 提出基于转接光顺的加工轨迹生成算法。 可在满足伺服约束和误差要求的前提下, 保证加工 轨迹的速度和加速度光滑连续。

在刀具轨迹的智能生成过程中, 不仅要考虑刀 具轨迹的几何特征优化, 同时也考虑不同刀具轨迹 对机床加工性能的影响。SHI 等 ${ }^{[42]}$ 分析了刀具路径 中的起始点、终点、进给方向、加工轨迹半径等因 素, 在切削力和残余高度上的影响。 $\mathrm{TUNC}^{[43]}$ 分析 了五轴铣削过程中加工轨迹在粗加工、半精加工和 精加工不同阶段中的铣削力、铣削稳定性及表面残 留高度, 并提出对三个加工过程中刀具轨迹的智能 优化方法。结果表明, 通过对半精加工刀具路径的 改进, 可以改善精加工阶段刀具路径切削力的均匀 一致性，同时也可以节约 33\%的加工时间。

\section{2 加工后大数据知识的智能自学习}

大数据知识是指机床智能系统在运行过程中, 围绕全制造生命周期, 包含工艺过程、加工状态感 知过程、智能控制优化过程等流程所产生的海量的 工业大数据知识。机床系统产生的工业大数据知识 除具有一般大数据的特征外, 还具有时序性、强关
联性、准确性以及闭环性等特征。

传统的机床控制系统，由于数据的采集、储存 和共享等方面的诸多因素限制，所产生的数据信息 在经过使用后并未保存，导致数据中所蕴含的知识 价值未被充分利用。随着大数据和云端技术的成熟 发展与广泛运用, 机床智能控制系统中的数据经过 采集、预处理、清洗和关联整合等步骤后可以上传 到云端数据中心，形成基于工业大数据的知识库。 加工大数据知识(库)的丰富完善, 极大地拓展了传 统工业数据范围，同时也进一步促进了人工智能技 术在机床智能控制系统中的深度发展应用。

\subsection{1 机床大数据知识的智能关联建模}

机床大数据知识的应用, 首要步骤是建立特定 的大数据知识集。其中包括对采集数据建立相关联 的知识标签, 形成大数据集的智能知识关联模型。 带标签的大数据知识对于人工智能技术极为重要, 是众多识别、分析、训练等算法的关键基础。

现有机床数据采集模型通常仅与时域标签关 联, 而基于时域标签的大数据模型, 难以准确量化 描述机床当前的加工任务。例如，当采集数据发生 突然改变时, 可能是加工状态的异常, 也可能是加 工任务的改变, 数据改变的因素难以体现在时域标 签上。CHEN 等 ${ }^{[44]}$ 提出机床加工过程数据的指令域 标签智能关联建模方法, 如图 8 所示。通过指令域 的 $\mathrm{G}$ 代码等, 将机床加工中的感知信息、反馈采样 周期与工作任务、加工资源等数据建立相关联的实 时映射关系。该方法有以下三个突出的优点: (1) 机 床内部的数据信息, 特别是指令信息, 可以被充分 理解和运用, 如 $\mathrm{G}$ 代码指令、主轴电流指令、进给 速度指令、插补指令等; (2) 通过对指令域中的工作 任务数据, 制造资源数据和操作状态数据等解析, 可以实时准确的表达输入变量和输出变量之间的关

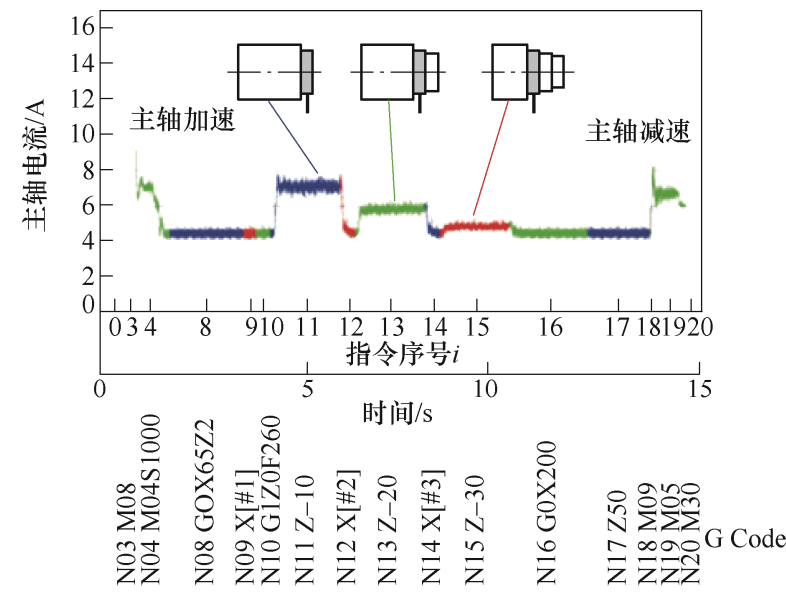

图 8 机床主轴电流数据的指令域标签智能关联 ${ }^{[44]}$ 
系, 并进行智能关联形成带知识标签的机床大数据 模型; (3) 随着对机床大数据知识的不断积累和更 新, 机床的理论数据模型可以随着时间的推移, 动 态的不断改进和进一步发展。

机床的大数据知识包括结构化、半结构化和非 结构化多种不同关系类型的数据。例如, 使用行列 等二维形式表示的结构化数据, 使用 XML 等表示 的半结构化数据, 以及几何信息等非结构化数据。 LIU 等 ${ }^{[45]}$ 针对机床系统中的几何信息、工艺信息和 监测信息等多源异构数据, 提出了涉及静态信息和 动态信息的有效同步关联方法。通过分析理论的离 散刀具点位置和实际采样的刀具点位置, 建立三种 信息之间的实时同步关联关系，同时还将时序匹配 和坐标匹配相结合以减少误匹配。试验表明刀位点 数据的匹配平均延迟小于 $0.1 \mathrm{~ms}$ 。针对机床大数据 在建立过程中存在的实时数据与非实时数据关联问 题。MENG 等 ${ }^{\left[{ }^{46]}\right.}$ 提出一种不同协议方式的模块化数 据通信和同步采集方法。该方法突出特点是使用开 放式的模块化架构, 因此不依赖于特定的机床或数 据采集设备，具有较好的通用性。

\subsection{2 机床大数据知识的智能分析}

机床大数据知识的分析与研究, 其本质就是从 复杂的机床数据中发现新的关联模式与知识, 通过 关联分析、聚类分析、融合分析等不同的智能分析 方法, 对大数据知识进行规律寻找及规律表示, 挖 掘获得有利用价值信息的过程 ${ }^{[47]}$ 。利用不同的机床 大数据知识集组合, 可以对机床的多个方面状态进 行智能分析与优化应用。

在机床加工过程的大数据知识分析及优化方 面, $\mathrm{JI}$ 等 ${ }^{[48]}$ 对机床的工件数据、刀具数据、机床数 据、加工要求、加工环境、加工结果等 60000 条大 数据, 通过结合进化算法(EA)、人工神经网络(ANN)、 层次分析法(AHP), 三种方法来智能分析和全局整 体优化加工中的机床选择、刀具选择以及加工参数。 该方法的机床大数据虽然使用 Matlab 随机生成, 还 不能对机床大数据知识进行标签化关联建模。但突 出的优点是将机床实际的加工环境转化为数学上的 大数据知识模型, 使得机床选择、刀具选择以及参 数选择等加工描述, 可以通过数学解析方法进行大 数据智能分析。同时, 通过对机床全局工艺的智能 大数据分析, 改进并优化了传统分布式工艺规划的 流程。

在机床刀具状态的大数据知识智能分析方面, ZHU 等 ${ }^{[49]}$ 提出面向大数据分析的智能刀具状态监 测系统框架, 如图 9 所示。针对机床加工中反馈的
力信号、振动信号、温度信号、声发射信号、2D 图 像、3D 点云数据、工艺参数等大数据知识, 利用深 度学习方法进行异构大数据融合及特征提取分析, 通过大数据智能分析刀具当前的磨损状态，而且可 以对刀具进行剩余寿命的预测和磨损补偿。该大数 据分析框架的突出优点, 是可以较全面的融合分析 多种刀具状态相关的大数据知识，同时也可以进一 步对采集的数据进行降噪和清洗, 提高大数据分析 结果的准确性。

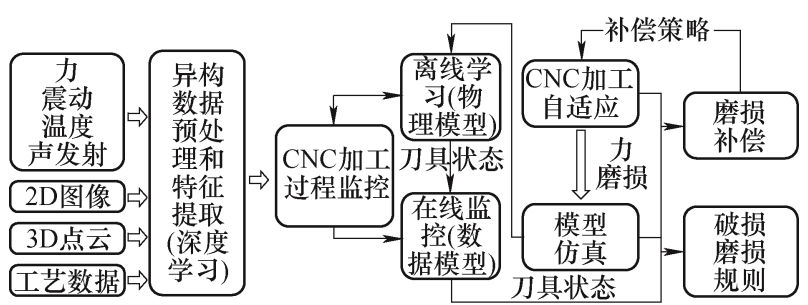

图 9 面向大数据的智能刀具监测框架 ${ }^{[48]}$

机床的大数据知识的深入研究, 已经广泛应用 于机床的各个方面分析, 如机床能耗方面的大数据 知识智能分析、机床故障诊断方面的大数据挖掘等。 随着机床大数据知识的丰富及智能分析技术的成 熟, 将能够得到更多的潜在加工规律，同时也为人 工智能技术提供更多的知识和数据支持。

\subsection{3 机床大数据知识的自学习成长}

机床大数据知识与人工智能技术深度结合, 不 仅可以保存并且复用已有的大数据知识, 而且通过 对神经网络等算法的数据模型不断积累和训练, 能 使机床的知识模型得以智能优化和自学习成长。

目前, 机床大数据知识的智能自学习成长, 已 经在机床的工艺大数据模型 ${ }^{[50]}$ 、加工状态监控大数 据模型 ${ }^{[51]}$ 、参数优化大数据模型 ${ }^{[52]}$ 、故障诊断大数 据模型 ${ }^{[33-54]}$ 等许多方面得到广泛应用, 并产生关键 性影响。以机床工艺方面的大数据模型为例, 知识 模型的自学习成长，使得工艺规划的基本架构模式 发生重要变化。工艺人员的设计规划不再局限于重 复的思考、重组和运用工艺规则。以人工智能技术 和大数据技术为核心的知识自学习成长模型, 将代 替人的经验进行大量的知识重用模拟与数据的推理 演算, 从而使工程师从大量的基础工作中解放出来, 极大地提升了设计人员的工作效率。

LI 等 ${ }^{[5]}$ 提出一种基于历史工艺知识重构的加 工特征定义方法。该方法对没有标签的历史工艺的 大数据知识进行分析和挖掘, 识别和重构加工特征, 然后对工艺特征和几何参数进行两次无监督的聚 类，自学习和积累工艺特征。虽然该方法前期需要 人工规划一些典型的零件工艺, 以完成初始的工艺 
大数据知识学习和积累。但随着历史工艺数据的不 断增多, 工艺知识系统可以不断地自学习和累积成 长。LIU 等 ${ }^{[56]}$ 基于数字孪生技术提出一种工艺知识 的重用和评价方法。该方法建立了工艺知识的大数 据知识模型和数字孪生模型。通过相似性计算从工 艺知识候选集中输出相似的工艺特征, 然后对输出 的工艺知识进行评价, 推导出最佳的工艺知识重用 方法。

机床的大数据知识模型研究至今, 都存在一个 普遍的矛盾: 机床的知识模型既要有高度的兼容性, 又要有针对的精确性。而机床大数据知识的自学习 成长可以很好地解决这一矛盾。泛用性强但不精确 的大数据知识模型, 可以通过不断的自学习成长, 变得越来越精确。同时, 这是优势也是弊端, 如何 保证机床的大数据知识模型在复杂的加工环境中稳 定、健康的成长, 是未来研究中急需解决的问题。

\section{4 机床智能控制系统的数字孪生技术}

在机械加工中, 由于机床切削过程的复杂特性, 智能系统需要分析和评估当前切削、刀具、工件、 机床等多个状态的实时变化, 结合数据监测手段, 形成机床智能系统的数字孪生模型, 对理论存在的 偏差进行感知与修正 ${ }^{[57]}$ 。

\section{1 刀具状态的数字孪生}

刀具状态是指机床在切削加工过程中, 刀具可 能发生的磨损、崩刃、断裂、变形等不同的状态。 目前已有很多学者对刀具状态的监测进行了深入的 研究 ${ }^{[58]}$, 通过切削过程的切削力、振动、声发射、 切削温度、主轴功率或电流、表面粗粘度、2D 图像、 $3 \mathrm{D}$ 点云数据等信息, 都可以实现刀具状态的感知及 数字孪生。

因为刀具的磨损在加工过程中是不可避免的, 因此在刀具状态的数字孪生中, 针对刀具磨损状态 的数字孪生应用最为广泛。刀具磨损会导致刀具的 性能质量随时间一直发生退化, 对机床的控制系统 产生切削力增大、切削温度升高、加工振动及工件 质量下降等负面影响。STAVROPOULOS 等 ${ }^{[59]}$ 通过 加速度和主轴电流数据, 将刀具磨损程度归类为高、 中、低三个水平进行预测。WANG 等 ${ }^{[60]}$ 通过加工参 数、主轴功率图、刀具磨损图等异构数据, 提出混 合机器学习方法来监测刀具磨损状态, 如图 10 所 示。并通过 H13 和 IN718 模具钢铣削试验, 证明该 方法的正确率超过 $90 \%$ 。HUANG 等 ${ }^{[61]}$ 分别从铣削 力、振动等多传感器信号中, 通过深度卷积神经网
络(DCNN)提取并融合时域、频域和时频域等多域特 征, 作为刀具磨损预测方法。

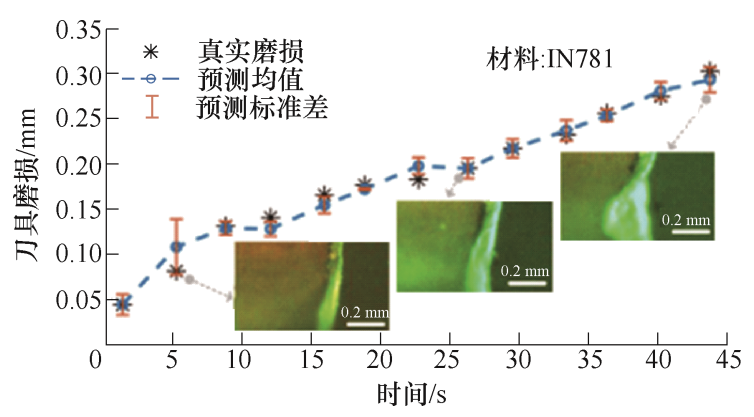

图 10 使用训练过的 CNN 识别磨损过程 ${ }^{[60]}$

在机床智能系统长期运行时, 布置过多的传感 器会显著地提高监测成本, 而且不便于高效率加工。 因此, 许多学者提出基于无传感器的刀具状态数字 孪生方法。例如, 在刀具的崩刃状态的数字孪生方 面, KANEKO 等 ${ }^{[62]}$ 通过结合传统切削力模型和主轴 扭矩信息, 改进了切削力的获取方式。提出一种不 需要铣削实验和其他外部力传感器, 即可监测机床 刀具的崩刀状态方法。在刀具的断裂状态数字孪生 方面, $\mathrm{XU}$ 等 ${ }^{[63]}$ 基于主轴电流和指令等机床内部数 据, 提出一种增量代价敏感型支持向量机(ICSSVM) 刀具破损监测方法。该方法比批量代价敏感型支持 向量机(BCSSVM)具有更高的效率。

另外, 刀具在其他方面状态的数字孪生也有许 多应用。针对刀具剩余寿命方面的数字孪生监测, JAIN 等 ${ }^{[64]}$ 提出一种基于加工质量与刀具退化关系 的刀具状态综合监测系统。该方法通过加工质量与 刀具退化的正相关关系，使用多级退化分类提高判 断的准确性, 并将刀具临界区作为刀具寿命的函数, 提高预测能力。针对其他刀具信息的数字孪生, BOTKINA 等 ${ }^{[65]}$ 使用国际 ISO 13399 标准, 设计了 刀具数字孪生体数据格式和结构, 信息流和数据管 理新信息流及数据孪生方法。

\section{2 工件状态的数字孪生}

工件状态的数字孪生是指在机床加工过程中, 对工件的几何精度、表面形貌、粗糙度、残余应力、 加工硬化等不同状态, 进行分析判断和数字孪生 建模。

针对工件的几何精度状态分析的判断和数字孪 生建模, ZHOU 等 ${ }^{[66]}$ 提出用插补点云色谱图(CPCI) 的新方法来描述工件的几何精度状态(图 11)。该方 法根据进给速度, 给每个插补点位分配一个颜色, 量化的描述了工件几何精度状态。同时还提出三角 面重建方法, 消除了相邻刀具路径上, 因进给速率 不连续而引起的表面加工缺陷。 


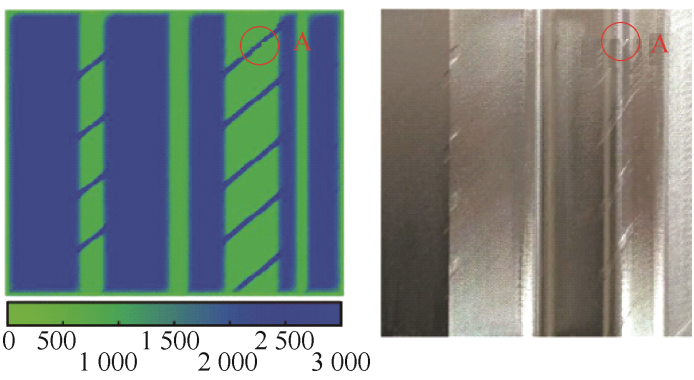

图 11 进给速度色谱图及实际工件状态 ${ }^{[66]}$

针对工件的表面粗䊁度的分析判断和数字孪生 建模, HUANG 等 ${ }^{[67]}$ 通过灰色理论和双边最佳拟合 方法, 揭示了铣削力信号与工件表面粗糙度的关系, 并提出表面粗䊁度灰色在线监测系统(GOMSRM)。 该系统的特点是仅需要较少的采样数据, 并且无须 训练时间即可在线预测工件的表面粗粘度。WU 等 ${ }^{[68]}$ 通过包络分析和统计计算提取振动信号的时 域特征和频域特征, 提出基于人工神经网络的工件 表面粗粘度预测方法。

针对工件残余应力的状态分析判断和数字孪生 建模, MA 等 ${ }^{[69]}$ 分析了热和机械载荷对残余应力形 成过程的影响, 并通过对不同进给速度和切削深度 下残余应力的分析, 提出了一种新的工件残余应力 预测模型, 该模型可以在端铣削过程中测量切削力 和切削温度。针对工件在加工过程中形变的状态分 析判断和数字孪生建模, 黄晓明等 ${ }^{[70]}$ 通过综合分析 工件刚度演变、应力状态、工件变形机制, 提出加 工变形数学模型和快速预测方法。

\section{3 机床状态的数字孪生}

机床的状态包括机床的加工误差、模态、刚度 等机床性能状态, 以及可能发生各种故障的异常状 态。机床状态的数字孪生是指上述对加工产生影响 的各种机床状态进行自主的建模分析, 从而为机床 智能控制系统的进一步控制和改进提供依据 ${ }^{[71]}$ 。

机床的加工误差是影响加工精度的主要因素, 加工误差涵盖几何误差 ${ }^{[72]}$ 、热误差 ${ }^{[73]}$ 、动态误差 ${ }^{[74]}$ 等多种因素。针对机床的几何误差建模, 项四通 等 ${ }^{[75]}$ 使用螺旋理论对机床运动学正逆解进行建模, 并通过引入误差扭曲概念分析和补偿 41 项与位置 无关和位置相关的几何误差(图 12), 提出五轴机床 几何误差的建模和补偿方法。董泽园等 ${ }^{[76]}$ 分析了三 轴机床的 18 项和 21 项几何误差模型, 通过对比建 模过程和几何误差的检测、辨识、补偿的仿真及试 验研究, 系统性的分析比较两种几何误差模型在实 际机床误差补偿过程中的有效性, 并指出 18 项误差 模型对描述三轴数控机床的全几何误差项具有较高 的精确度和简洁性。

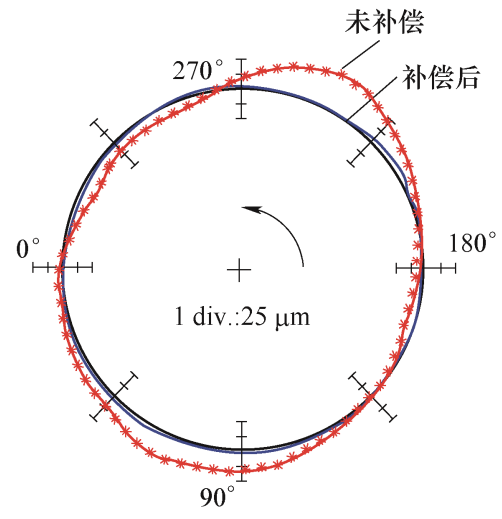

图 12 C 轴误差补偿结果对比 ${ }^{[75]}$

针对机床的热误差建模，刘阔等 ${ }^{[77]}$ 提出一种无 传感器的机床进给轴热误差补偿方法。该模型可以 在恒温环境下计算半闭环进给轴因运动生热导致的 热误差。由于无温度传感器, 因此该方法对机床的 启停、运动速度、运动范围等具有很强的鲁棒性。 李涁等 ${ }^{[78]}$ 在建立机床热误差模型时, 使用遗传算法 优化小波神经网络的权值因子, 来提高机床热误差 模型的预测精度、鲁棒性及收玫性。

针对驱动跟踪误差及丝杜反向间隙误差等运动 误差建模, LI 等 ${ }^{[79]}$ 使用深度置信网络建立了机床反 向间隙误差预测模型，并将反向间隙误差判断和预 测结果与 BP 神经网络和支持向量机(SVM)预测方 法进行对比，表明 DBN 方法在预测方面的显著的 优势。JIANG 等 ${ }^{[80]}$ 提出一种非线性时间序列建模方 法，以增强机床进给系统误差的预测准确性。该方 法首先使用朴素贝叶斯方法(NB-MSC)对机床的启 动状态、变速状态、稳定状态、反向状态、停止状 态等五个状态进行分类，然后通过非线性自回归长 短记忆网络(NAR-LSTM)建立三种状态的子模型, 对进给系统的运动误差进行建模分析和预测。该方 法的突出优点是采用运动分类的方法细分了机床的 误差模型，使得机床进给系统的误差预测精度大大 提高。

针对机床刚度、模态等动态特性建模, 吴石 等 ${ }^{[81]}$ 分析了机床在不同的加工姿态下，机床横梁、 刀柄、刀具、工件曲面特征等对加工系统综合刚度 性能的影响, 并建立了机床加工系统的综合刚度性 能云图。该方法突出的优点是将机床的刚度与被加 工曲面关联, 可以清晰地描述出工件被加工区域的 综合刚度。王伟等 ${ }^{[82]}$ 分析了机床刚度对加工轮廓的 影响机理。通过计算弹性变形, 将机床的动力学模 型与加工轮廓误差关联起来。杨闪闪等 ${ }^{[83}$ 提出一种 基于理论的五轴数控机床空间动态特性分析方法。 该方法首先使用分层拉丁超立方方法, 对机床的固 
有频率进行采样, 然后通过径向基函数理论, 插值 建立出机床在不同姿态下的动态特性模型。

\section{4 切削状态的数字孪生}

切削状态包括空切、稳定切削、不稳定切削和 其他异常状态等 ${ }^{[84]}$ 。通过对切削状态的数字孪生建 模分析, 可以提高机床智能系统的感知能力和稳 定性。

稳定切削状态的数字孪生, 是指对稳定加工过 程中的切削力、振动、温度、能耗、噪声等多种状 态指标进行的建模分析。在切削力的建模分析方面, YAMATO $^{[85]}$ 和 ALTINTAS ${ }^{[86]}$ 等均通过建立机床进 给系统的驱动力传导方程的方法(图 13), 提出使用 进给驱动器的电流数据, 来预测机床在加工过程中 铣削力的方法。

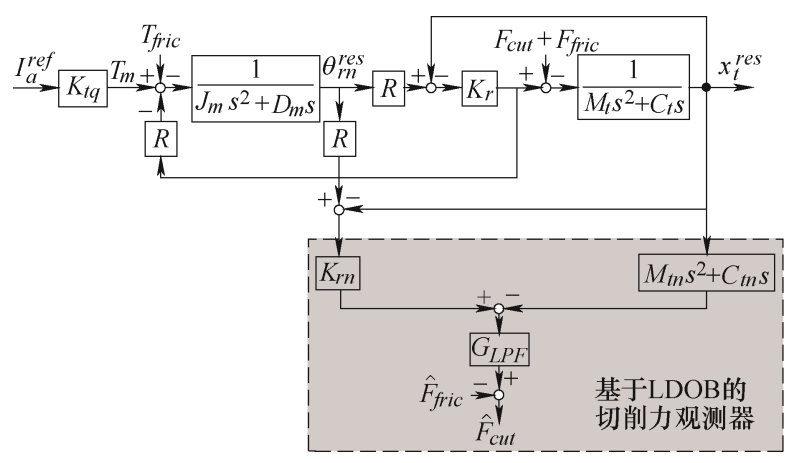

图 13 基于 $\mathrm{LDOB}$ 的切削力观测模型 ${ }^{[85}$

在切削温度建模分析方面, NING 等 ${ }^{[87]}$ 提出一 种快速预测正交切削中两个变形区(主剪切区和刀 具一切屑界面)加工温度的分析方法。通过计算切削 力和温度的关联性, 结合切削方程和材料本构方程, 建立了切削温度的原始分析及预测模型。岳彩旭 等 ${ }^{[88}$ 基于 “移动热源法” 提出一种铣刀前刀面瞬态 温度场的建模方法。通过对第一变形区和第二变形 区的建模分析和叠加计算, 并试验验证了瞬态温度 场模型的有效性。

在加工能耗的建模分析方面, MENG 等 ${ }^{[89-90]}$ 基于塑性变形理论和摩擦理论, 从能量守恒角度提 出了机床加工过程中的能耗建模方法。通过分析主 轴转速、进给速度、切宽、切深等因素, 分类细化 并建立了线性单元、圆弧单元以及棱柱等不同特征 的切削能耗模型。

不稳定切削状态主要是指对切削过程中发生的 颤振等不稳定状态进行建模分析。YANG 等 ${ }^{[91}$ 提出 了一种基于振动信号自动分析铣削状态的建模方 法。该方法使用深度置信神经网络学习振动信号特 征, 同时结合无监督贪婪分层关联和有监督微调的 两个训练步骤, 可以自动的提取和分类振动信号的
特征。最终通过投票策略识别机床空切、稳定切削 和颤振三种铣削状态。

为保证机床在连续加工过程中的工件质量, 针 对连续加工过程中可能发生的断刀、严重磨损、主 轴失效等其他异常状态, LIANG 等 ${ }^{[92]}$ 提出一种数据 驱动的加工过程异常诊断方法。通过对加工过程的 功率数据进行采集、降噪、标准化和校对等预处理, 以及基于数据的绝对平均值、峰值以及峰值因子矩 阵等, 来表示数据的关键特征。最后, 通过关键特 征与阈值的对比, 判断加工是否处于异常状态。该 方法的优点是异常状态的判断阈值可以不依赖于人 工经验设定, 而且判断阈值可以根据历史数据, 用 果蝇优化算法进行动态调整。

可以看到, 国内外学者采用不同物理信号、不 同的智能理论及预测模型，对机床、工件、刀具及 加工状态的监控、分析、预测, 取得了丰硕的成果, 为数字孪生理论与方法研究奠定了坚实的基础。但 目前数字孪生研究工作中, 多数以其他条件恒定不 变为前提, 忽略了机床智能控制系统的全局综合性 影响。学者在数字孪生研究工作中也更多关注于分 析结果的准确性或计算效率。对数字孪生模型的兼 容性, 是否便于融入全局机床智能控制系统等方面 的考虑较少。

\section{5 机床智能控制系统的云服务技术}

随着制造业由粗放型向精益型转变, 客户需求 导向开始贯穿整个生产流程, 实体价值和服务价值 同等重要。生产者不再仅满足于销售机床本身，而 是更注重于机床的全生命周期服务, 如数据分析、 生产培训、订单管理、机床维护升级等。同时, 消 费者也不仅满足于购买机床产品, 而是更关注于机 床及其独有的加工技术与服务, 为其带来的直接效 益。而云服务技术就是负责机床智能控制系统的业 务环节, 是机床智能控制系统的对外网络窗口。云 服务通过连接入工厂、企业等服务网络, 可以极大 地拓展机床智能控制系统的业务服务范围。

\section{1 云端智能设计服务}

工艺技术一直是各生产加工行业的关注热点。 每年生产企业都需要招收大量人员以满足加工设计 需求。但这些工艺技术却很少以价值的形式流通, 最终形成技术孤岛。将工艺设计作为一种云服务资 源进行推广, 可以加速工艺技术的流通和完善。利 用云端平台提供低成本、高质量的智能设计服务, 具有较高的可行性和较好的发展潜力。 
在云端智能设计服务方面, 胡天亮等人研究了 云端工艺知识库的搭建方法 ${ }^{[93]}$ 、云端工艺知识库中 工艺知识的智能查询及推理机制 ${ }^{[94]}$ 、以及 $\mathrm{CNC}$ 中 使用云端知识库进行工艺的智能规划方法 ${ }^{[95]}$, 对云 端工艺知识的智能设计服务全流程进行了较为全面 的探索和研究。该学者首先将工艺相关知识从 STEP-NC 映射为网络本体语言(WOL), 然后通过 Hadoop 平台将工艺知识存储到 Hbase 数据库中, 并 基于 MapReduce 框架研发出工艺知识的查询推理引 擎, 最后在 $\mathrm{CNC}$ 中加载云端数据库并进行数据交 互, 完成从云端到 $\mathrm{CNC}$ 本地端的工艺技术智能云设 计服务的应用方法综合研究。

基于云知识的工艺设计服务, 需要服务供应商 前期投入大量的研发资金, 用于实验不同工艺参数 对加工工件的质量影响。因此, 多数工艺服务供应 商不愿意在云端公布或分享核心工艺知识。针对此 问题, 李迎光等研究了云端设计服务中工艺知识的 封装方法 ${ }^{[96]}$ 以及复杂自由曲面工艺的云端智能设 计服务方法 ${ }^{[97]}$, 如图 14 所示。通过标准化的加工 任务描述策略, 将加工服务封装在每个服务提供商 中, 并提出一种新的云制造服务框架以及工艺知识 封装方法。该框架在云端仅将服务供应商的可执行 功能信息提供给客户, 因此可以避免云服务供应商 的核心工艺知识泄漏。同时基于表面动态加工特征 (SDMF) 的概念, 提出复杂自由曲面加工的云端智能 工艺设计方法。该方法基于 SDMF-FB 的事件驱动 机制, 可以使加工服务自适应响应加工资源的变化, 从而获取加工表面的最佳加工工艺。

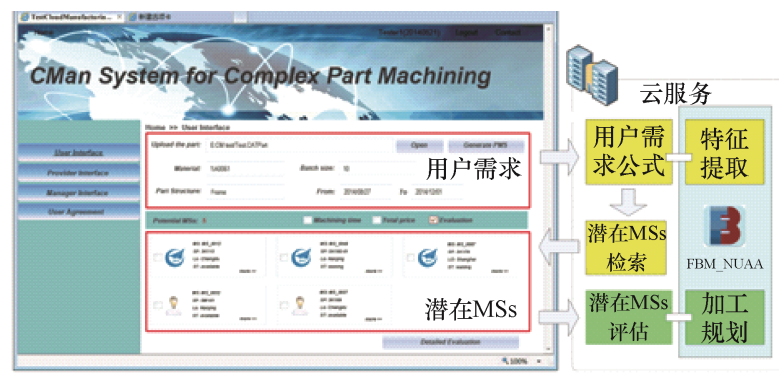

图 14 云端智能工艺设计服务 ${ }^{[96]}$

\section{2 云端智能数据服务}

云端智能数据服务包括对机床的加工数据采 集、存储、分析、优化及共享等数据方面的智能云 服务。徐旬等 ${ }^{[98-99]}$ 通过定义机床数据的对象、变量、 方法和从属关系, 建立了符合 OPC-UA 标准的机床 泛用数据模型, 并使用 MTConnect 协议, 提高了数 据采集的应用兼容性。WANG 等 ${ }^{[100]}$ 建立了较为全 面的切削数据云平台系统。该平台借助多传感器和
分布式存储技术, 搭建了数据采集层和数据存储层。 通过数学建模和数据挖掘算法建立了信息的分析与 处理层。最后通过云服务器提供智能的知识查询、 推理和优化, 建立智能操作服务层。建立的切削数 据云平台系统，可在云系统中实现对切削数据的多 源采集、快速访问和存储、智能建模与分析、高效 挖掘与智能优化等功能, 弥补和完善了当前云端智 能切削数据库系统的平台架构, 是较为综合有效的 切削大数据云智能服务方法。

\section{3 云端智能控制与运算服务}

机床厂商和客户期望机床的控制系统具有良好 的知识计算能力和远程控制能力、易于维护、具有 良好的兼容性以及可扩展性。当前的数控系统由于 硬件的限制难以达到上述要求，但云端智能控制与 运算服务可以很好地解决这些问题。

徐句等 ${ }^{[101]}$ 基于 “控制系统即服务(CSaaS)” 的 云服务思想, 提出了一种基于云端的数控系统方案。 CSaaS 通过分离机床的控制系统的实时任务与非实 时任务的方法，将对机床的非实时控制转化成为基 于云的服务。同时，该方法还研究了运动控制数据 的传输机制, 以减少传输延迟导致的控制系统错误。 杨建中等 ${ }^{[102]}$ 基于 “前端即服务(FAAS)” 的云服务 思想, 提出了一种基于云计算的机床控制系统方案, 如图 15 所示。通过将传统数控系统的前端控制器转 化为云服务, 不仅可以通过多种设备访问数控系统, 而且还可以兼容第三方软件并提供更强的计算能力。

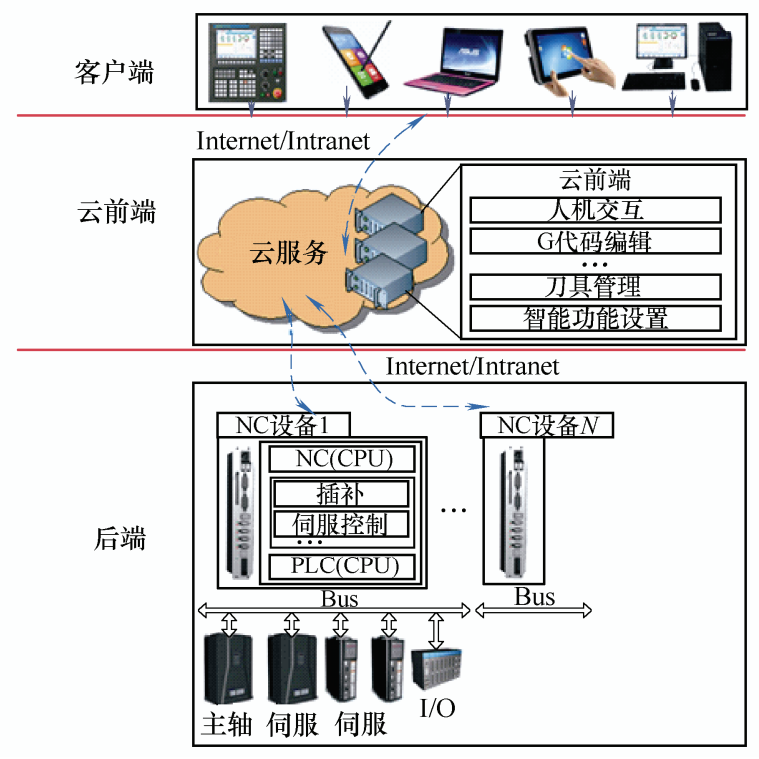

图 15 基于云服务的数控系统架构 ${ }^{[102]}$

云端智能控制与运算服务不仅可以增强单机系 统的控制与运算能力, 还可以对车间生产线中的多 
个设备在云端进行集群在线控制。富宏亚和刘健康 等 ${ }^{[103]}$ 研究了面向集群部署的数控系统微服务架构。 基于 Linux 实时扩展内核提出了生产线中数控机床 集群的集中在线控制方法。该方法通过统一的工业 实时以太网协议, 解决了生产线中多个数控机床以 及工业机器人的通讯兼容问题。使用 Apache Spark 框架搭建系统的数据库和计算引擎, 并通过 Docker 和共享内存技术解决了不同操作系统的控制冲突和 协作交互问题。

\section{4 云端智能维护服务}

云端智能维护服务是保证机床及生产线长时 间、稳定运行的基础, 对降低机床异常故障、提高 加工质量的稳定性等具有重要意义。目前云端维护 服务包括机床的状态监测、产品良率分析、故障警 报、远程诊断等技术支持, 但在机床性能退化诊断、 机床潜在故障预测等方面仍具有进一步发展空间。

针对机床的性能退化以及刀具磨损等隐性异常 的累积分析, 及智能诊断维护问题, ZHANG 等 ${ }^{[104]}$ 基于离散制造车间中的能源大数据, 提出一种机床 隐性生产异常的检测方法。通过布置能耗传感器和 读取 RFID 标签中的工艺流程来采集能耗数据, 并 将其输入到深度置信神经网络中用以训练和分析。 该方法对蜗杆生产车间的车床、铣床和蜗杆磨床进 行四个半月的能耗数据采集试验。最终结果表明, 预测准确率可达 96.32\%。 KUMARA 等 ${ }^{[105]}$ 基于 MapReduce 大数据框架, 提出一种在云服务环境下 自动识别故障的诊断方法。该方法的突出优点是可 以通过支持向量机 (SVM) 径向基函数插值和 SMOTE 分类方法, 解决实际生产过程中因故障发 生少, 而导致的数据不平衡问题, 如图 16 所示。

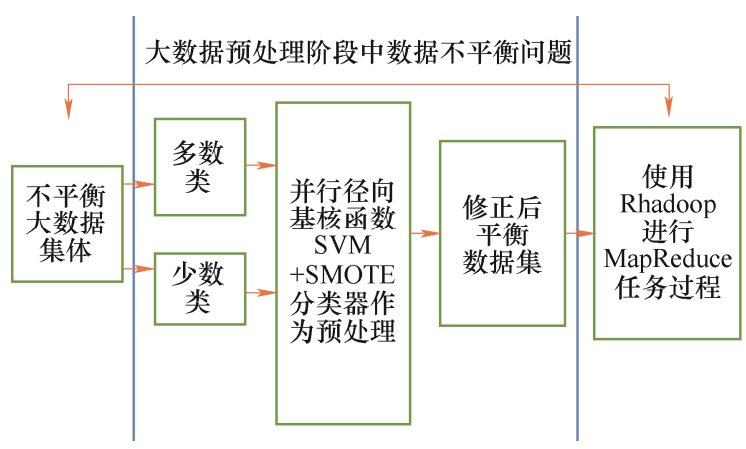

图 16 针对大数据集不平衡问题的预处理流程 ${ }^{[05]}$

近年来, 云服务的发展方向可以归纳为以下几 个方面: 基于制造的云服务、基于产品的云服务、 基于过程的云服务、面向市场的云服务等。机床现 有的云服务应用包括: 生产订单交易、设备租赁、 机床状态采集、大数据展示、加工任务管理、生产
效能统计、故障警报分析, 以及远程技术诊断等方 面的云服务。但在产品的全生命周期跟踪管理、加 工工艺的创新与优化、加工知识的智能分析与推荐、 加工过程的直接远程控制, 以及生产价值的互联互 通等云服务方面的应用较少, 仍具有较大的提升 空间。

\section{6 结论与展望}

智能制造已成为世界各国制造业发展的共同主 题。随着新一代的智能技术革新, 机床智能控制系 统也发展到了新的阶段。作为战略性新兴产业的智 能高档数控机床, 在可以预计的未来将面临激烈的 竞争，同时也面临着新的机遇和挑战。

(1) 机床智能控制系统的承载功能范围面临着 新的机遇和挑战。如今的机床智能控制系统不再仅 仅局限于承担加工职能, 而是兼容涵盖了向设计、 仿真、感知、分析、控制、维护、诊断等多功能一 体化的方向发展。

(2) 机床智能控制系统的兼容性面临着新的机 遇和挑战。随着机床智能控制系统开放性的逐步提 升, 以及机床数字孪生和接口协议的标准化, 未来 的机床智能系统将有能力与多种上下游协作软件、 不同品牌的加工协作设备、外接部件等设备, 进行 多方面的无缝兼容及协作。

(3) 机床智能控制系统的感知与分析能力面临 着新的机遇和挑战。如今机床智能控制系统已被海 量的数据所包围: 如切削力、振动、声音、温度、 位置、图像、能耗、几何信息、工艺参数、内部控 制指令等多个方面的海量数据。随着系统的开放性 增加, 以往机床中的 “黑匣子” 数据也可以被提取 分析。因此, 如何高效地分析与利用这些数据, 必 然是未来发展的研究热点。

(4) 机床智能控制系统的控制方式面临着新的 机遇和挑战。机床的控制技术发展就是机器逐步代 替人的过程。人工智能技术的丰富及 $\mathrm{AI}$ 芯片技术的 发展, 使得用户控制难度正在逐步降低。未来将不 仅只有用户单独思考控制机床, 而是用户与 $\mathrm{AI}$ 一起 合作完成控制任务。

(5) 机床智能控制系统中的应用程序正面临云 服务升级挑战。如今机床的本体设备已经不再仅能 搭载单一厂商的操作系统或应用程序。互联网技术 的升级和云服务技术的完善, 极大地改善了机床中 应用程序的研发难度和推广环境。如何利用这些优 势, 提供低成本、高质量的应用程序, 是今后机床 
应用程序服务中需要考量的重要因素。

(6) 机床智能控制系统的技术研发面临着新的 机遇和挑战。机床智能控制系统的技术类别繁多、 覆盖面广泛, 以往机床企业 “单打独斗” 的研发模 式, 已经难以跟上机床新技术的扩张速度。如何建 立起机床系统的技术联盟, 结合产、学、研等联合 发展, 解决现有的机床企业局面、建立良性发展循 环, 是未来机床技术研发中亟须解决的关键难题。

\section{参 考 文 献}

[1] KANG H S, LEE J Y, CHOI S S, et al. Smart manufacturing: Past research, present findings, and future directions $[\mathrm{J}]$. International Journal of Precision Engineering and Manufacturing-green Technology, 2016, 3(1): 111-128.

[2] ZHONG R Y, XU X, KLOTZ E, et al. Intelligent manufacturing in the context of industry 4.0: A review[J]. Engineering, 2017, 3(5): 616-630.

[3] 王磊, 卢秉恒. 中国工作母机产业发展研究[J]. 中国工 程科学, 2020, 22(2): 29-37.

WANG Lei, LU Bingheng. Research on the development of machine tool industry in China[J]. Strategic Study of CAE, 2020, 22(2): 29-37.

[4] WANG B. The future of manufacturing : A new perspective[J]. Engineering, 2018, 4(5): 722-728.

[5] XU L D, XU E L, LI L. Industry 4.0: State of the art and future trends[J]. International Journal of Production Research, 2018, 56(8): 2941-2962.

[6] 张曙. 智能制造与 i5 智能机床 $[\mathrm{J}]$. 机械制造与自动化, 2017, 46(1): 1-8.

ZHANG Shu. Smart manufacturing and i5 smart machine tools [J]. Machine Building \& Automation, 2017, 46(1): $1-8$.

[7] TAO F, QI Q, LIU A, et al. Data-driven smart manufacturing[J]. Journal of Manufacturing Systems, 2018, 48: 157-169.

[8] ALCÁCER V, CRUZ-MACHADO V. Scanning the industry 4.0: A literature review on technologies for manufacturing systems[J]. Engineering Science And Technology-An International Journal-Jestech, 2019, 22(3): 899-919

[9] ZHOU J, LI P, ZHOU Y, et al. Toward new-generation intelligent manufacturing[J]. Engineering, 2018, 4(1): 11-20.

[10] WANG L. From intelligence science to intelligent manufacturing[J]. Engineering, 2019， 5(4): 615-618.

[11] 刘强. 智能制造理论体系架构研究 [J]. 中国机械工程, 2020, 31(1): 24-36.
LIU Qiang. Study on achitecture of intelligent manufacturing theory $[\mathrm{J}]$. China Mechanical Engineering, 2020, 31(1): 24-36.

[12] 叶佩青, 张勇, 张辉. 数控技术发展状况及策略综述 $[\mathrm{J}]$. 机械工程学报, 2015, 51(21): 113-120.

YE Peiqing, ZHANG Yong, ZHANG Hui. Review on the development and strategies of CNC technology[J]. Journal of Mechanical Engineering，2015，51(21): 113-120.

[13] 刘献礼, 刘强, 岳彩旭, 等. 切削过程中的智能技术 [J]. 机械工程学报, 2018, 54(16): 45-61.

LIU Xianli, LIU Qiang, YUE Caixu, et al. Intelligent machining technology in cutting process [J]. Journal of Mechanical Engineering, 2018，54(16): 45-61.

[14] 黄莹, 卢秉恒, 赵万华. 云计算在智能机床控制体系中 的应用探析 [J]. 机械工程学报, 2018, 54(8): 210-216. HUANG Ying, LU Bingheng, ZHAO Wanhua. Application of cloud computing in intelligent machine tool control system[J]. Journal of Mechanical Engineering, 2018, 54(8): 210-216.

[15] CHEN J, HU P, ZHOU H, et al. Toward intelligent machine tool[J]. Engineering, 2019, 5(4): 679-90.

[16] XU X W, NEWMAN S T. Making CNC machine tools more open, interoperable and intelligent $-\mathrm{a}$ review of the technologies[J]. Computers in Industry, 2006, 57(2): 141-152.

[17] ESMAEILIAN B, BEHDAD S, WANG B. The evolution and future of manufacturing: A review[J]. Journal of Manufacturing Systems, 2016, 39: 79-100.

[18] WANG S, WAN J, ZHANG D, et al. Towards smart factory for industry 4.0: A self-organized multi-agent system with big data based feedback and coordination[J]. Computer Networks, 2016, 101: 158-168.

[19] ATZORI L, IERA A, MORABITO G. The internet of things: A survey[J]. Computer Networks, 2010, 54(15): 2787-2805.

[20] LU Y, XU X. Cloud-based manufacturing equipment and big data analytics to enable on-demand manufacturing services $[\mathrm{J}]$. Robotics and Computer-Integrated Manufacturing, 2019, 57: 92-102.

[21] LIU Y, WANG L, WANG X V, et al. A revisit to cloud manufacturing[J]. China Mechanical Engineering, 2018, 29(18): 2226-2237.

[22] XU X. Machine tool 4.0 for the new era of manufacturing $[\mathrm{J}]$. The International Journal of Advanced Manufacturing Technology, 2017, 92(5-8): 1893-1900.

[23] SHARP M, AK R, HEDBERG Jr T. A survey of the advancing use and development of machine learning in smart manufacturing[J]. Journal of manufacturing systems, 2018, 48: 170-179. 
[24] LI B, HOU B, YU W, et al. Applications of artificial intelligence in intelligent manufacturing: A review[J]. Frontiers of Information Technology \& Electronic Engineering, 2017, 18(1): 86-96.

[25] TAO F, QI Q, WANG L, et al. Digital twins and cyber-physical systems toward smart manufacturing and Industry 4.0: correlation and comparison[J]. Engineering, 2019, 5(4): 653-661.

[26] QI Q, TAO F. Digital twin and big data towards smart manufacturing and industry 4.0:360 degree comparison[J]. IEEE Access, 2018， 6: 3585-3593.

[27] TALKHESTANI B A, JUNG T, LINDEMANN B, et al. An architecture of an intelligent digital twin in a cyber-physical production system[J]. At Automatisierungstechnik, 2019, 67(9): 762-782.

[28] HU L, NGUYEN N T, TAO W, et al. Modeling of cloud-based digital twins for smart manufacturing with MT connect[J]. Procedia Manufacturing, 2018, 26 : 1193-1203.

[29] 陶飞, 戚庆林. 面向服务的智能制造 $[\mathrm{J}]$. 机械工程学 报，2018，54(16): 11-23.

TAO Fei , QI Qinglin. Service-oriented smart manufacturing [J]. Journal of Mechanical Engineering, 2018, 54(16): 11-23.

[30] WANG J, MA Y, ZHANG L, et al. Deep learning for smart manufacturing: Methods and applications[J]. Journal of Manufacturing Systems, 2018， 48: 144-156.

[31] KIM D H, KIM T J Y, WANG X, et al. Smart machining process using machine learning: A review and perspective on machining industry[J]. International Journal of Precision Engineering and Manufacturing-Green Technology, 2018, 5(4): 555-568.

[32] KUMAR S P L. State of the art-intense review on artificial intelligence systems application in process planning and manufacturing $[\mathrm{J}]$. Engineering Applications of Artificial Intelligence, 2017，65: 294-329.

[33] JING X, ZHU Y, LIU J, et al. Intelligent generation method of 3D machining process based on process knowledge[J]. International Journal of Computer Integrated Manufacturing, 2020, 33(1): 38-61.

[34] 张禹, 李东升, 董小野, 等. 基于改进 BP 神经网络面 向 STEP-NC 2.5D 制造特征的智能宏观工艺规划[J]. 机 械工程学报, 2020，56(1): 148-156.

ZHANG Yu, LI Dongsheng, DONG Xiaoye, et al. The intelligent macro-process planning based on an improved BP neural network for STEP-NC 2.5D manufacturing features[J]. Journal of Mechanical Engineering, 2020, 56(1): 148-156.

[35] JI W, WANG L, HAGHIGHI A, et al. A reachability based approach for machining feature sequencing $[\mathrm{J}]$. Journal of Manufacturing Systems, 2016, 40: 96-104.

[36] LIU C, LI Y, SHEN W. An adaptive process planning method based on Features and intelligent agents for the manufacturing of large-scale parts[J]. IFAC-Papers on Line, 2015, 48(3): 2214-2219.

[37] TUNC L T, OZKIRIMLI O M, BUDAK E. Machining strategy development and parameter selection in 5-axis milling based on process simulations $[\mathrm{J}]$. The International Journal of Advanced Manufacturing Technology, 2016, 85(5-8): 1483-1500.

[38] 㚞文刚, 叶佩青. 复杂曲面五轴端铣加工刀具轨迹规划 研究进展 $[\mathrm{J}]$. 机械工程学报, 2015, 51(15): 168-182.

FAN Wengang, YE Peiqing. Research progress in tool path planning for five-axis end milling machining of sculptured surfaces[J]. Journal of Mechanical Engineering, 2015, 51(15): 168-182.

[39] CHEN C S, SUN Y T A. Intelligent computer-aided process planning of multi-axis $\mathrm{CNC}$ tapping machine $[\mathrm{J}]$. IEEE Access， 2017， 5: 2913-2920.

[40] KARUPPANAN B R C , SARAVANAN M . Optimized sequencing of $\mathrm{CNC}$ milling toolpath segments using metaheuristic algorithms[J]. Journal of Mechanical Science and Technology, 2019，33(2): 791-800.

[41] 赵欢, 张永红, 丁汉. 五轴线性刀路的转接光顺及轨迹 生成算法[J]. 机械工程学报, 2018, 54(3): 108-116.

ZHAO Huan, ZHANG Yonghong, DING Han. A corner rounding and trajectory generation algorithm for five-axis linear toolpath [J]. Journal of Mechanical Engineering, 2018, 54(3): 108-116.

[42] SHI K, LIU N, WANG S, et al. Effect of tool path on cutting force in end milling $[\mathrm{J}]$. The International Journal of Advanced Manufacturing Technology , 2019 , 104(9-12): 4289-4300.

[43] TUNC L T. Smart tool path generation for 5-axis ball-end milling of sculptured surfaces using process models[J]. Robotics and Computer-Integrated Manufacturing, 2019, 56: $212-221$.

[44] CHEN J, YANG J, ZHOU H, et al. CPS modeling of CNC machine tool work processes using an instruction-domain based approach[J]. Engineering, 2015, 1(2): 247-260.

[45] LIU C, LI Y, WANG Q, et al. A synchronous association approach of geometry, process and monitoring information for intelligent manufacturing $[\mathrm{J}]$. Robotics and Computer-Integrated Manufacturing, 2019, 58: 120-129.

[46] MENG Boyang, LI Maoyue, LIU Xianli, et al. Open architecture $\mathrm{CNC}$ system based on soft-integrated communication[J]. Procedia CIRP, 2018， 72: 671-676.

[47] SHANG C, YOU F. Data analytics and machine learning 
for smart process manufacturing: Recent advances and perspectives in the big data era[J]. Engineering, 2019, 5(6): 1010-1016.

[48] JI W, YIN S, WANG L. A big data analytics based machining optimisation approach[J]. Journal of Intelligent Manufacturing, 2019, 30(3): 1483-1495.

[49] ZHU K, LI G, ZHANG Y. Big data oriented smart tool condition monitoring system[J]. IEEE Transactions on Industrial Informatics, 2020, 16(6): 4007-4016.

[50] LI X, ZHANG S, HUANG R, et al. A survey of knowledge representation methods and applications in machining process planning[J]. The International Journal of Advanced Manufacturing Technology, 2018, 98(9-12): 3041-3059.

[51] ZHU K, ZHANG Y. A cyber-physical production system framework of smart CNC machining monitoring system[J]. IEEE/ASME Transactions on Mechatronics, 2018, 23(6): 2579-2586.

[52] CHANG W Y, WU S J. Big data analysis of a mini three-axis $\mathrm{CNC}$ machine tool based on the tuning operation of controller parameters $[\mathrm{J}]$. The International Journal of Advanced Manufacturing Technology, 2018, 99(5-8): 1077-1083.

[53] SU Y, TAO F, JIN J, et al. Failure prognosis of complex equipment with multistream deep recurrent neural network[J]. Journal of Computing and Information Science in Engineering, 2020， 20(2): 021007.

[54] CHIU M C, TSAI C D, LI T L. An integrative machine learning method to improve fault detection and productivity performance in a cyber-physical system[J]. Journal of Computing and Information Science in Engineering, 2020, 20(2): 021009.

[55] LIU C, LI Y, LI Z. A machining feature definition approach by using two-times unsupervised clustering based on historical data for process knowledge reuse[J]. Journal of Manufacturing Systems， 2018，49: 16-24.

[56] LIU J, ZHOU H, TIAN G, et al. Digital twin-based process reuse and evaluation approach for smart process planning[J]. The International Journal of Advanced Manufacturing Technology, 2019, 100(5-8): 1619-1634.

[57] ZHOU G, ZHANG C, LI Z, et al. Knowledge-driven digital twin manufacturing cell towards intelligent manufacturing $[\mathrm{J}]$. International Journal of Production Research, 2019: 1-18.

[58] ZHOU Y, XUE W. Review of tool condition monitoring methods in milling processes[J]. The International Journal of Advanced Manufacturing Technology, 2018, 96(5-8): 2509-2523.

[59] STAVROPOULOS P, PAPACHARALAMPOPOULOS
A, VASILIADIS E, et al. Tool wear predictability estimation in milling based on multi-sensorial data[J]. The International Journal of Advanced Manufacturing Technology, 2016, 82(1-4): 509-521.

[60] WANG P, LIU Z, GAO R X, et al. Heterogeneous data-driven hybrid machine learning for tool condition prognosis[J]. CIRP Annals, 2019， 68(1): 455-458.

[61] HUANG Z, ZHU J, LEI J, et al. Tool wear predicting based on multi-domain feature fusion by deep convolutional neural network in milling operations $[\mathrm{J}]$. Journal of Intelligent Manufacturing, 2019: 1-14.

[62] KANEKO K, NISHIDA I, SATO R, et al. Machining state monitoring in end milling based on comparison of monitored and predicted cutting torques[J]. Journal of Advanced Mechanical Design , Systems , and Manufacturing, 2019, 13(3): 1-11.

[63] XU G, ZHOU H, CHEN J. CNC internal data based incremental cost-sensitive support vector machine method for tool breakage monitoring in end milling $[\mathrm{J}]$. Engineering Applications of Artificial Intelligence, 2018, 74: 90-103.

[64] JAIN A K, LAD B K. A novel integrated tool condition monitoring system[J]. Journal of Intelligent Manufacturing, 2019, 30(3): 1423-1436.

[65] BOTKINA D, HEDLIND M, OLSSON B, et al. Digital twin of a cutting tool[J]. Procedia CIRP, 2018, 72: 215-218.

[66] ZHOU H, LANG M, HU P, et al. The modeling, analysis, and application of the in-process machining data for $\mathrm{CNC}$ machining $[\mathrm{J}]$. The International Journal of Advanced Manufacturing Technology, 2019, 102(5-8): 1051-1066.

[67] HUANG P T B, ZHANG H J, LIN Y C. Development of a grey online modeling surface roughness monitoring system in end milling operations[J]. Journal of Intelligent Manufacturing, 2019, 30(4): 1923-1936.

[68] WU T Y, LEI K W. Prediction of surface roughness in milling process using vibration signal analysis and artificial neural network[J]. The International Journal of Advanced Manufacturing Technology, 2019, 102(1-4): 305-314.

[69] MA Y, FENG P, ZHANG J, et al. Prediction of surface residual stress after end milling based on cutting force and temperature[J]. Journal of Materials Processing Technology, 2016, 235: 41-48.

[70] 黄晓明, 孙杰, 李剑峰. 基于刚度与应力演变机制的航 空整体结构件加工变形预测理论建模 [J]. 机械工程学 报，2017，53(9): 201-208.

HUANG Xiaoming, SUN Jie, LI Jianfeng. Mathematical modeling of aeronautical monolithic component 
machining distortion based on stiffness and residual stress evolvement [J]. Journal of Mechanical Engineering, 2017, 53(9): 201-208.

[71] LUO W , HU T, ZHANG C , et al. Digital twin for CNC machine tool: Modeling and using strategy[J]. Journal of Ambient Intelligence and Humanized Computing, 2019, 10(3): 1129-1140.

[72] 李杰, 谢福贵, 刘辛军, 等. 五轴数控机床空间定位精 度改善方法研究现状 [J]. 机械工程学报, 2017, 53(7): 113-128

LI Jie, XIE Fugui, LIU Xinjun, et al. Analysis on the research status of volumetric positioning accuracy improvement methods for five-axis NC machine tools [J]. Journal of Mechanical Engineering, 2017, 53(7): 113-128.

[73] 王海同, 李铁民, 王立平, 等. 机床热误差建模研究综 述[J]. 机械工程学报, 2015, 51(9): 119-128.

WANG Haitong, LI Tiemin, WANG Liping, et al. Review on thermal error modeling of machine tools[J]. Journal of Mechanical Engineering, 2015, 51(9): 119-128.

[74] LYU D, LIU Q, LIU H, et al. Dynamic error of CNC machine tools : A state-of-the-art review[J]. The International Journal of Advanced Manufacturing Technology, 2020, 106(5-6): 1869-1891.

[75] XIANG S, ALTINTAS Y. Modeling and compensation of volumetric errors for five-axis machine tools[J]. International Journal of Machine Tools and Manufacture, 2016, 101: 65-78.

[76] 董泽园, 李杰, 刘辛军, 等. 数控机床两种几何误差建 模方法有效性试验研究[J]. 机械工程学报, 2019, 55(5): 137-147.

DONG Zeyuan, LI Jie, LIU Xinjun, et al. Experimental study on the effectiveness of two different geometric error modeling methods for machine tools[J]. Journal of Mechanical Engineering, 2019， 55(5): 137-147.

[77] 刘阔, 孙名佳, 吴玉亮, 等. 无温度传感器的数控机床 进给轴热误差补偿 $[\mathrm{J}]$. 机械工程学报, 2016, 52(15): 162-169.

LIU Kuo, SUN Mingjia, WU Yuliang, et al. Thermal error compensation without temperature sensors for cnc machine tools' feed drive system[J]. Journal of Mechanical Engineering, 2016, 52(15): 162-169.

[78] 李涁, 张云, 王立平, 等. 基于遗传算法优化小波神经 网络数控机床热误差建模 [J]. 机械工程学报, 2019, 55(21): 215-220.

LI Bin, ZHANG Yun, WANG Liping, et al. Modeling for CNC machine tool thermal error based on genetic algorithm optimization wavelet neural networks [J]. Journal of Mechanical Engineering, 2019, 55(21): 215-220.
[79] LI Z, WANG Y, WANG K. A data-driven method based on deep belief networks for backlash error prediction in machining centers[J]. Journal of Intelligent Manufacturing, 2017: 1-13.

[80] JIANG Y, CHEN J, ZHOU H, et al. Nonlinear time-series modeling of feed drive system based on motion states classification[J]. Journal of Intelligent Manufacturing, 2020: $1-14$

[81] 吴石, 李荣义, 刘献礼, 等. 复杂曲面模具加工系统综 合刚度场建模与分析[J]. 机械工程学报, 2016, 52(23): 189-198.

WU Shi, LI Rongyi, LIU Xianli, et al. The modeling and analysis about comprehensive stiffness field of complex curved surface mould processing system [J]. Journal of Mechanical Engineering, 2016， 52(23): 189-198.

[82] 王伟, 张心羽, 梅雄. 五轴数控机床进给系统刚度对自 由曲面轮廓误差影响机理研究 $[\mathrm{J}]$. 机械工程学报, 2016, 52(21): 146-154.

WANG Wei, ZHANG Xinyu, MEI Xiong. Research on the mechanism of free surface contour error caused by the stiffness of feed system of five-axis machine tools [J]. Journal of Mechanical Engineering, 2016, 52(21): 146-154

[83] 杨闪闪, 王玲, 廖启豪, 等. 基于径向基函数法的五轴 数控机床空间动态性能研究[J]. 机械工程学报, 2019, 55(9): 144-153.

YANG Shanshan, WANG Ling, LIAO Qihao, et al. Study on the spatial dynamic performance of five-axis nc machine tool based on radial basis function method[J]. Journal of Mechanical Engineering, 2019, 55(9): 144-153.

[84] YUE Caixu, GAO Haining, LIU Xianli, et al. A review of chatter vibration research in milling[J]. Chinese Journal of Aeronautics, 2019, 32(2): 215-242.

[85] YAMATO S, SUGIYAMA A, SUZUKI $\mathrm{N}$, et al. Enhancement of cutting force observer by identification of position and force-amplitude dependent model parameters[J]. The International Journal of Advanced Manufacturing Technology, 2019, 104(9-12): 3589-3605.

[86] ASLAN D, ALTINTAS Y. Prediction of cutting forces in five-axis milling using feed drive current measurements[J]. IEEE/ASME Transactions on Mechatronics, 2018, 23(2): 833-844

[87] NING J, LIANG S Y. Predictive modeling of machining temperatures with force-temperature correlation using cutting mechanics and constitutive relation[J]. Materials, 2019, 12(2): 284.

[88] 岳彩旭, 都建标, 刘献礼, 等. 考虑时变性热强度和时 变性热量分配比的铣刀前刀面瞬态温度场建模研究 [J]. 机械工程学报，2019，55(9)：206-216. 
YUE Caixu, DU Jianbiao, LIU Xianli, et al. Modeling research on transient temperature field of rake face on end mills considering time-varying heat intensity and time-varying distribution ratio [J]. Journal of Aeronautics, 2019, 55(9): 206-216.

[89] MENG $\mathrm{Y}$, WANG $\mathrm{L}$, LEE $\mathrm{C} \mathrm{H}$, et al. Plastic deformation-based energy consumption modelling for machining[J]. The International Journal of Advanced Manufacturing Technology, 2018, 96(1-4): 631-641.

[90] WANG L, MENG Y, JI W, et al. Cutting energy consumption modelling for prismatic machining features[J]. The International Journal of Advanced Manufacturing Technology, 2019, 103(5-8): 1657-1667.

[91] FU Y, ZHANG Y, GAO H, et al. Automatic feature constructing from vibration signals for machining state monitoring $[\mathrm{J}]$. Journal of Intelligent Manufacturing, 2019, 30(3): 995-1008.

[92] LIANG Y C, WANG S, LI W D, et al. Data-driven anomaly diagnosis for machining processes[J]. Engineering, 2019, 5(4): 646-652.

[93] YE Y, HU T, ZHANG C, et al. Design and development of a CNC machining process knowledge base using cloud technology $[\mathrm{J}]$. The International Journal of Advanced Manufacturing Technology, 2018, 94(9-12): 3413-3425.

[94] YANG Y, HU T, YE Y, et al. A knowledge generation mechanism of machining process planning using cloud technology $[\mathrm{J}]$. Journal of Ambient Intelligence and Humanized Computing, 2019, 10(3): 1081-1092.

[95] YE Y, HU T, YANG Y, et al. A knowledge based intelligent process planning method for controller of computer numerical control machine tools[J]. Journal of Intelligent Manufacturing, 2018: 1-17.

[96] LIU X, LI Y, WANG L. A cloud manufacturing architecture for complex parts machining[J]. Journal of Manufacturing Science and Engineering, 2015, 137(6).

[97] LIU X, LI Y. Feature-based adaptive machining for complex freeform surfaces under cloud environment[J]. Robotics and Computer-Integrated Manufacturing, 2019,
56: $254-263$.

[98] LIU C, VENGAYIL H, LU Y, et al. A cyber-physical machine tools platform using OPC UA and MTConnect[J]. Journal of Manufacturing Systems, 2019， 51: 61-74.

[99] LIU C, VENGAYIL H, ZHONG R Y, et al. A systematic development method for cyber-physical machine tools[J]. Journal of Manufacturing Systems，2018，48: 13-24.

[100] WANG Z, JIAO L, YAN P, et al. Research and development of intelligent cutting database cloud platform system[J]. The International Journal of Advanced Manufacturing Technology, 2018，94(9-12): 3131-3143.

[101] SANG Z, XU X. The framework of a cloud-based CNC system[J]. Procedia CIRP, 2017，63: 82-88.

[102] HUI E, FENG B, LEE C, et al. A design of CNC architecture based on cloud computing $[\mathrm{J}]$. Proceedings of the Institution of Mechanical Engineers, Part B: Journal of Engineering Manufacture, 2019, 233(4): $1260-1268$.

[103] LIU J, FU H, JIN H. Design of a centralized intelligent control system architecture for nc machining production lines $[\mathrm{C}] / /$ Proceedings of the International Symposium on Flexible Automation, 2018: 183-186.

[104] ZHANG C , WANG Z , DING K , et al. An energy-aware cyber physical system for energy big data analysis and recessive production anomalies detection in discrete manufacturing workshops[J]. International Journal of Production Research, 2020(1): 1-19.

[105] KUMAR A, SHANKAR R, CHOUDHARY A, et al. A big data map reduce framework for fault diagnosis in cloud-based manufacturing $[\mathrm{J}]$. International Journal of Production Research, 2016， 54(23): 7060-7073.

作者简介: 孟博洋, 男, 1991 年出生, 博士研究生。主要研究方向为机 床智能控制系统、运动控制及智能加工技术。

E-mail: bymeng@hrbust.edu.cn

李茂月(通信作者), 男, 1981 年出生, 博士, 副教授, 博士研究生导师。 主要研究方向为开放式数控系统及智能加工技术等。

E-mail:1my0500@163.com 